\title{
Drug Molecule Diflunisal Forms Crystalline Inclusion Complexes with Multiple Types of Linear Polymers
}

\author{
Zhi Zhong, ${ }^{\dagger}$ Canxiong Guo,${ }^{\dagger}$ Xiaotong Yang, ${ }^{\dagger}$ Baohua Guo,${ }^{\dagger}$ Jun Xu,,${ }^{\dagger}$ and Yanbin \\ Huang*,
}

${ }^{\dagger}$ Key Laboratory of Advanced Materials (MOE), Department of Chemical Engineering, Tsinghua University, Beijing 100084, China; Tel: 86-10-62797572; E-mail: jun-xu@mail.tsinghua.edu.cn (J. X.),and yanbin@tsinghua.edu.cn (Y.H.).

${ }^{\ddagger}$ College of Science, Beijing University of Chemical Technology, Beijing 100029, China

\section{Materials}

Diflunisal (DIF) was purchased from Spectrum Chemical (98\% purity). Polytetrohydrofuran (PTHF, Mn 2900 Da), poly- $\varepsilon$-caprolactone (PCL, Mn 10000 Da) and poly(butylene adipate) (PBA, Mw 12000 Da) were obtained from Sigma-Aldrich. Oligomers (PTHF650 and PCLdiol530) used for growing single crystals were also purchased from Sigma-Aldrich. Biologically produced poly-4-hydroxybutyrate (P4HB, Mn $\sim 4000$ Da by GPC, with P3HB components) was kindly provided by Professor Tadahisa Iwata (University of Tokyo). All the solvents used were AP grade and obtained from InnoChem (China). All the materials were used without further purification.

\section{Preparation of DIF pure forms, DIF-chloroform solvate and DIF-polymer ICs}

(1) DIF pure forms and DIF-chloroform solvate

The commercial DIF was identified as Form I by PXRD. DIF Form II, Form III and the chloroform solvate were prepared following the methods described in the previous literature ${ }^{1}$.

(2) DIF-PTHF2900 IC

DIF (0.45 g) and PTHF2900 (0.06 g) were dissolved in ethanol $(30 \mathrm{ml})$, and stirred for $10 \mathrm{~min}$ at $45{ }^{\circ} \mathrm{C}$ to ensure complete dissolution. The obtained solution was then allowed to evaporate slowly at room temperature for 3 days until white crystals formed. The IC crystals were collected and gently washed 2-3 times with dichloromethane to remove the excess PTHF, and then dried 
under vacuum at room temperature for $24 \mathrm{~h}$.

(3) DIF-PCL10000 IC

DIF (0.45 g) and PCL10000 (0.06 g) were dissolved in a solvent mixture (4:1 ethyl acetate: heptane, total $40 \mathrm{ml}$ ), and stirred for $10 \mathrm{~min}$ at $45^{\circ} \mathrm{C}$ to ensure complete dissolution. The obtained solution was then allowed to evaporate slowly at room temperature for 3 days until white crystals formed. The IC crystals were collected and gently washed 2-3 times with dichloromethane to remove the excess PCL, and then dried under vacuum at room temperature for $24 \mathrm{~h}$.

\section{(4) DIF-P4HB4000 IC}

DIF (0.45 g) and P4HB4000 (0.06 g) were dissolved in acetone $(30 \mathrm{ml})$, and stirred for $10 \mathrm{~min}$ at $40{ }^{\circ} \mathrm{C}$ to ensure complete dissolution. The obtained solution was then filtered through a $0.22 \mu \mathrm{m}$ PVDF syringe filter to remove the insoluble impurities in the biologically produced raw material. The filtrate was collected and allowed to evaporate slowly for 3 days to form white crystals. The IC crystals were collected and gently washed 2-3 times with dichloromethane to remove the excess $\mathrm{P} 4 \mathrm{HB}$, and then dried under vacuum at room temperature for $24 \mathrm{~h}$.

(4) DIF-PBA12000 IC

DIF (0.45 g) and PBA12000 (0.06 g) were dissolved in ethyl acetate $(30 \mathrm{ml})$, and stirred for 10 min at $45^{\circ} \mathrm{C}$ to ensure complete dissolution. The obtained solution was then allowed to evaporate slowly at room temperature for 3 days until white crystals formed. The IC crystals were collected and gently washed 2-3 times with dichloromethane to remove the excess PBA, and then dried under vacuum at room temperature for $24 \mathrm{~h}$.

\section{Single crystal growth}

Single crystals of diffraction quality were obtained by crystallization from a DIF-saturated solution of DIF/PTHF650 or DIF/PCLdiol530 (DIF/polymer ratio 90/10, w/w) in ethyl acetate by slow vapor diffusion of hexane. Colorless, prism-shaped crystals were collected for the diffraction measurements and structural determination. 


\section{Characterization}

(1) Single crystal X-ray diffraction

Single crystals of DIF-PTHF $\left(0.500 \times 0.200 \times 0.120 \mathrm{~mm}^{3}\right)$ and DIF-PCL $(0.500 \times 0.220 \times$ $0.170 \mathrm{~mm}^{3}$ ) were used for the diffraction measurement. X-ray reflections were collected on a Bruker Smart APEX II CCD area detector using graphite-monochromated Mo $K \alpha$ radiation $(0.71073 \AA)$ at $100 \mathrm{~K}$. The crystal structures were solved by direct method using the SHELXS-97 program $^{2}$ and refined by full-matrix least-squares procedure using the SHELXL-2014 program ${ }^{3}$. Non-hydrogen atoms were refined anisotropically. All hydrogen atoms were generated geometrically. In these channel lattices, electron densities corresponding to the guest polymers were highly disordered and could not be accurately modeled. Hence, they were squeezed out from the structural models using PLANTO/SQUEEZE ${ }^{4}$. The stoichiometric ratios of DIF and guest polymers in these IC crystals were determined by ${ }^{1} \mathrm{H}-\mathrm{NMR}$, and the state of polymer chains was investigated by ssNMR.

(2) Powder X-ray diffraction (PXRD)

Samples for PXRD measurement were grinded manually with an agate mortar and pestle before test. Diffraction measurement with powders through 200 mesh screen were carried out at room temperature using a Rigaku D/max-2500 X-ray diffractometer with $\mathrm{Cu} K \alpha$ radiation (1.54056 $\AA$ ). Samples were placed on the sample holder which has $1 \mathrm{~mm}$ thickness and $1.5 \times 1.0 \mathrm{~cm}$ dimensions. Continuous scans were performed at a speed of $2^{\circ} 2 \theta / \min$ and $0.02^{\circ} 2 \theta$ per step in the range $2 \theta=$ $2-45^{\circ}$. The patterns obtained were then processed using the Jade software (version 6.0, Materials Data, Inc.).

\section{(3) Solid-state NMR (ssNMR)}

All solid-state NMR spectra were acquired using a Bruker AV 300 spectrometer operating at $75.4 \mathrm{MHz}$ for ${ }^{13} \mathrm{C}$ (7T static magnetic field). Samples were packed into a $4 \mathrm{~mm} \mathrm{ZrO}_{2}$ rotor and sealed with Kel-F caps. Experiments were performed using $4 \mathrm{~mm}$ double resonance MAS probe. ${ }^{13} \mathrm{C}$ spectra for different samples were acquired under magic angle spinning (MAS) at different frequencies: DIF Form I 6 kHz, Form II 6.5 kHz, Form III 6.5 kHz, DIF-chloroform $12 \mathrm{kHz}$, 
DIF-PTHF2900 6.5 kHz, DIF-PCL10000 6.5 kHz, DIF-P4HB4000 12 kHz, DIF-PBA12000 6kHz, PTHF2900 $6 \mathrm{kHz}$, PCL10000 $6 \mathrm{kHz}$, P4HB4000 $6 \mathrm{kHz}$, and PBA12000 $6 \mathrm{kHz}$. Ramped-amplitude CP and tppm decoupling were used for these spectra. A $3 \mathrm{~ms}$ contact time was used in experiments. Glycine was used as an external standard, with carbonyl resonance at 176.03 ppm. All experiments were conducted at room temperature.

(4) Solution ${ }^{1} \mathrm{H}-\mathrm{NMR}$

Solution ${ }^{1}$ H-NMR measurements were performed on a JOEL JNM-ECA300 nuclear magnetic resonance spectrometer. Deuterated acetone was used as the solvent. Analysis of the obtained spectra was performed using the MestReNova software (version 6.0.2, Mestrelab Research, S. L.).

(5) Thermogravimetric analysis (TGA)

TGA was performed on a TA Instruments TGA Q500IR thermogravimetric analyzer. 10-15 mg of the sample was added on a platinum pan. The samples were heated over the temperature range 25-300 ${ }^{\circ} \mathrm{C}$ for DIF pure forms, DIF solvate and DIF ICs, and $25-800{ }^{\circ} \mathrm{C}$ for polymers, at a constant heating rate of $10{ }^{\circ} \mathrm{C} / \mathrm{min}$. The samples were purged with a stream of flowing nitrogen at $50 \mathrm{ml} / \mathrm{min}$ throughout the experiment.

(6) Differential scanning calorimetry (DSC)

DSC analysis was conducted with a SHIMADZU DSC-60 differential scanning calorimeter. Temperature calibration was carried out using an indium metal standard supplied with the instrument. Powder samples were weighed out $(2-2.5 \mathrm{mg})$ in aluminum pans and analyzed from $-30{ }^{\circ} \mathrm{C}$ to $240{ }^{\circ} \mathrm{C}$ at heating and cooling rates both of $10{ }^{\circ} \mathrm{C} / \mathrm{min}$ using a similar empty pan as a reference. An inert atmosphere was maintained in the calorimeter by purging nitrogen gas at a flow rate of $50 \mathrm{ml} / \mathrm{min}$.

(7) Polarized Optical Microscope (POM)

Polarized optical microscope (BX41, Olympus) equipped with a digital camera (Moticam Pro 282A, Motic) and a hot stage (LTS420, Linkam), was used to observe the thermal behavior of DIF ICs at a heating rate of $10^{\circ} \mathrm{C} / \mathrm{min}$. 


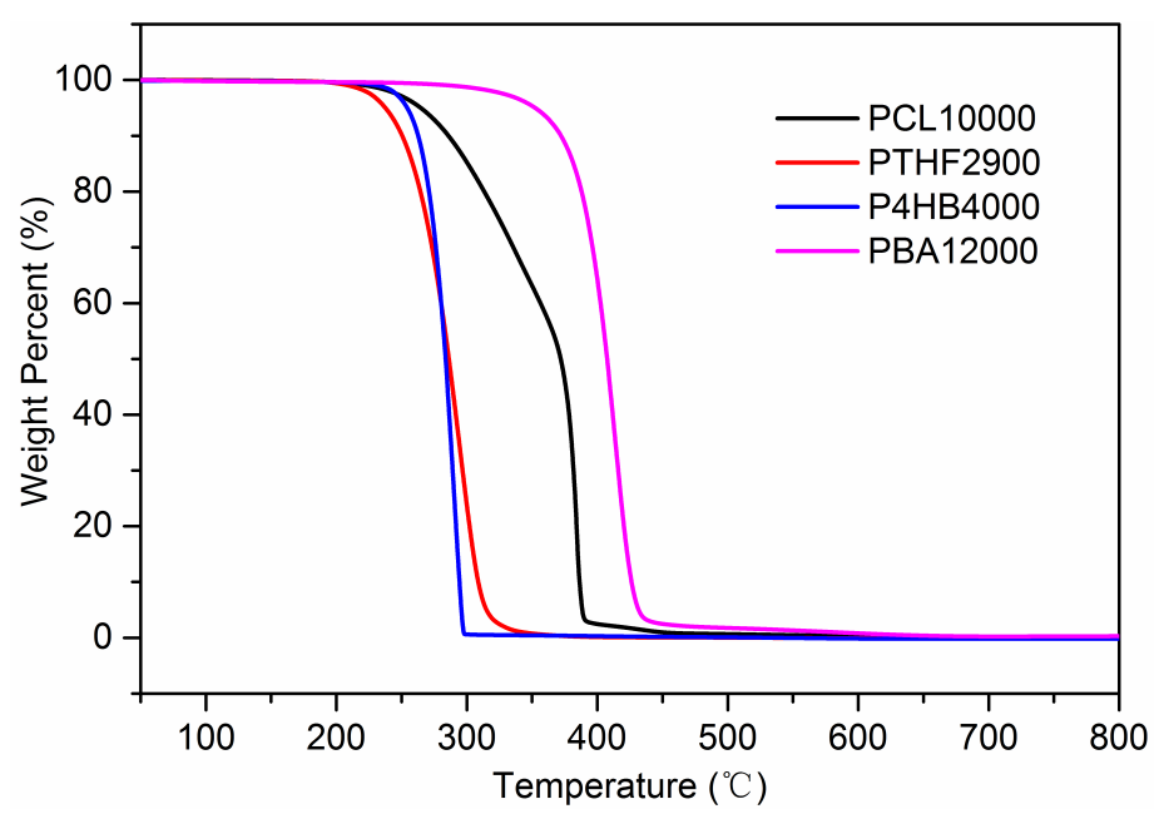

(a)

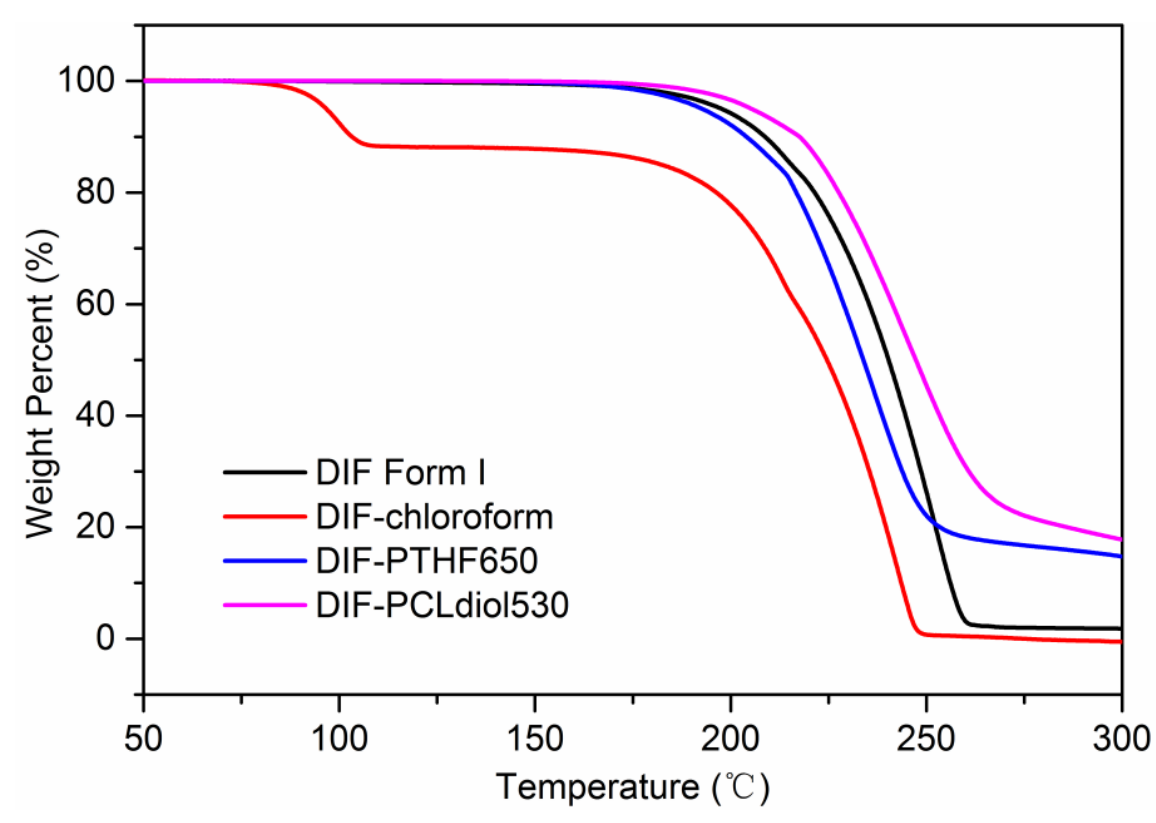

(b)

Figure S1. (a) TGA thermograms of the guest polymers measured at a heating rate of $10{ }^{\circ} \mathrm{C} / \mathrm{min}$. All the four polymers showed a significant weight loss only above $260^{\circ} \mathrm{C}$. (b) TGA thermograms of DIF-PTHF and DIF-PCL single crystal materials compared with those of DIF pure forms and the chloroform solvate. No detectable weight loss was observed for DIF-PTHF650 and DIF-PCLdiol530 below $150{ }^{\circ} \mathrm{C}$, indicating that no solvate was formed during the single crystal growth process. 


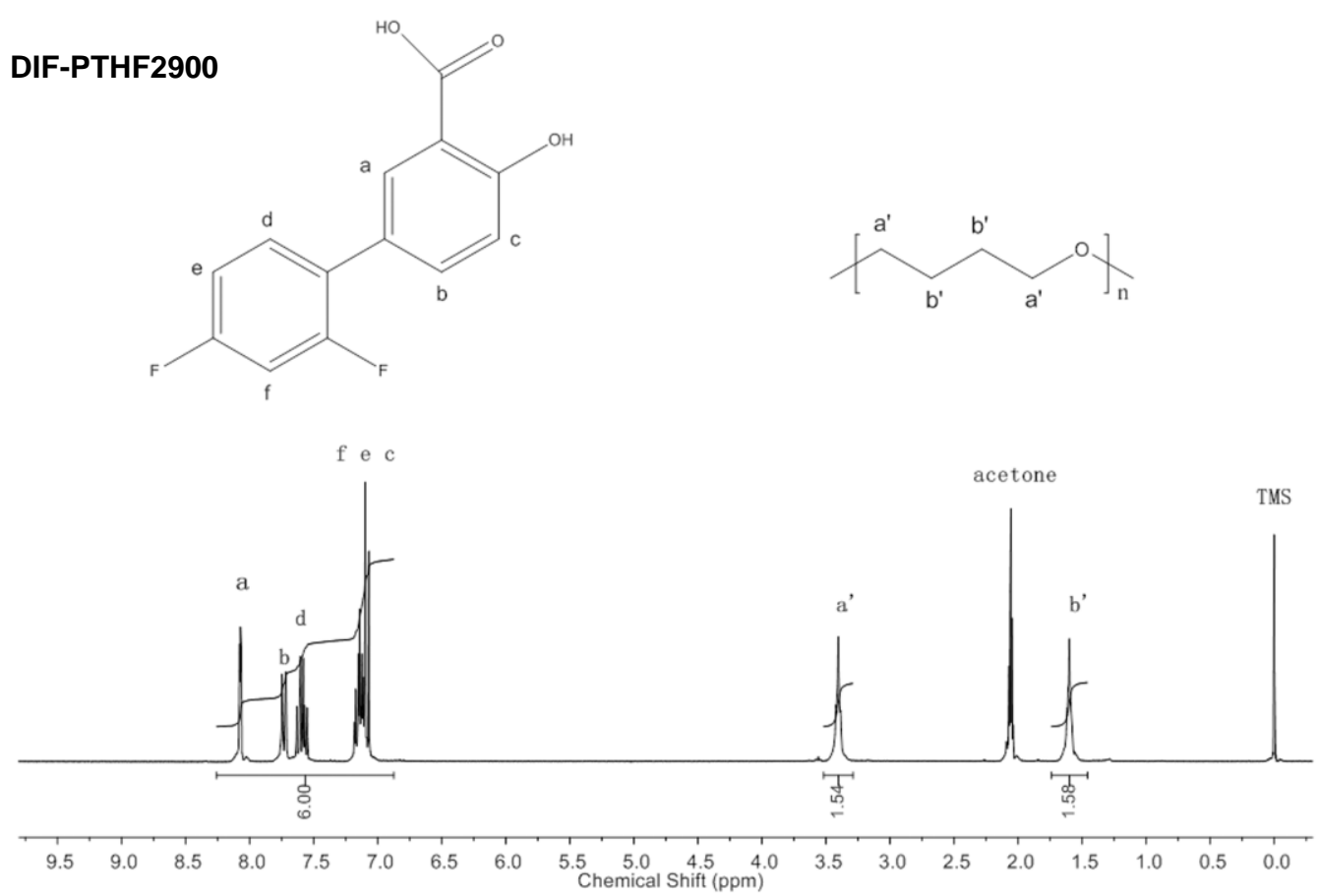

(a)

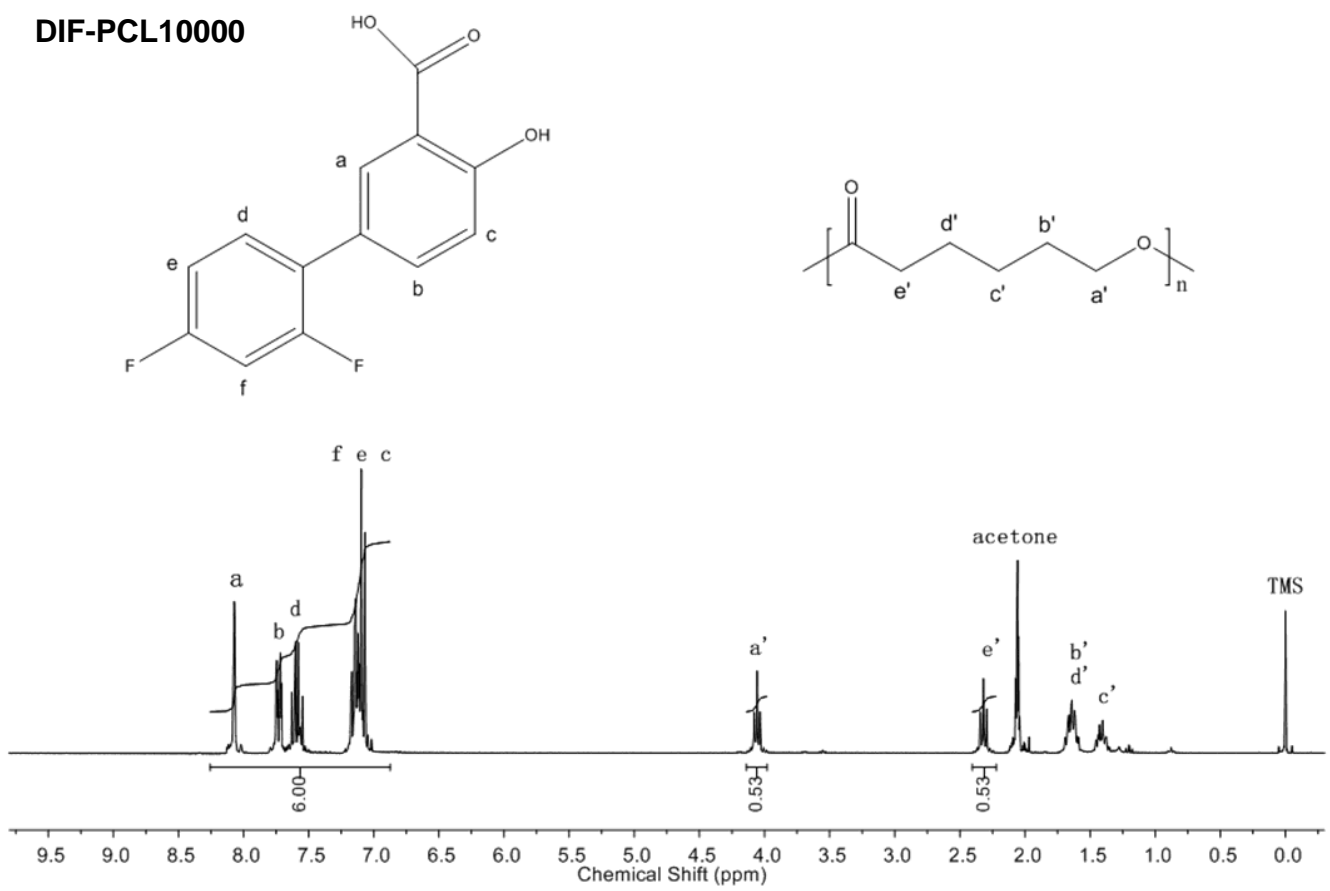

(b) 


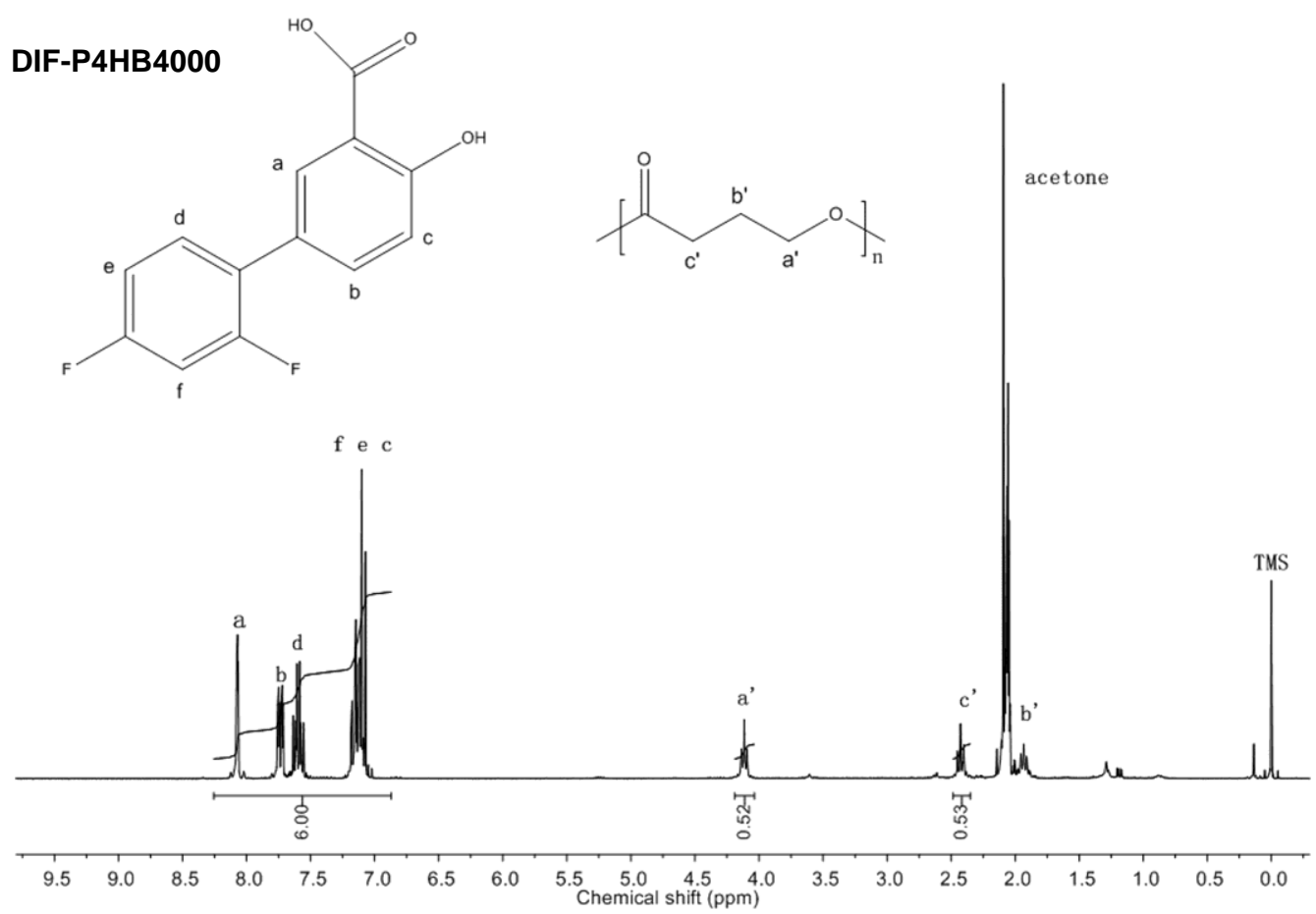

(c)

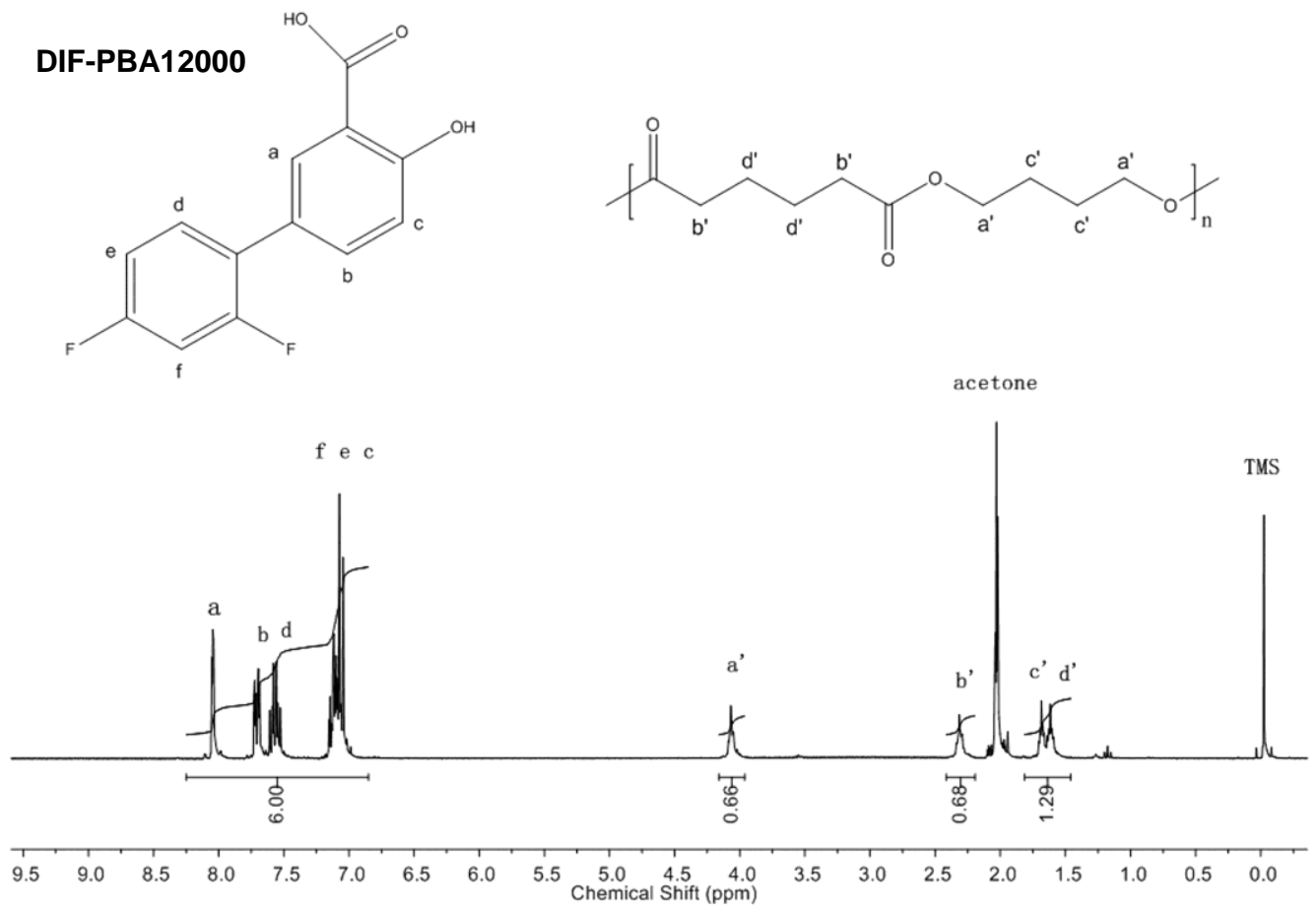

(d)

Figure S2. ${ }^{1}$ H-NMR spectra of (a) DIF-PTHF2900, (b) DIF-PCL10000, (c) DIF-P4HB4000 and (d) DIF-PBA12000 ICs. Acetone- $\mathrm{d}_{6}$ was used as the solvent. Characteristic peaks corresponding to the different protons in DIF and guest polymers are marked with corresponding letters. The 
stoichiometric ratios of DIF to monomers in different ICs were calculated using the integral area of the characteristic peaks. P4HB is a biologically produced polyester and other components (such as $\mathrm{P} 3 \mathrm{HB}$ ) existed in the raw materials, as evidenced by the resonance peaks which do not belong to P4HB (highlighted by the red wireframes in the following figure). Therefore the accurate DIF: monomer ratio in DIF-P4HB IC cannot be determined in this study due to the inclusion of other guest components in the DIF channels.

\section{P4HB raw material}<smiles>COCCCC(C)=O</smiles>

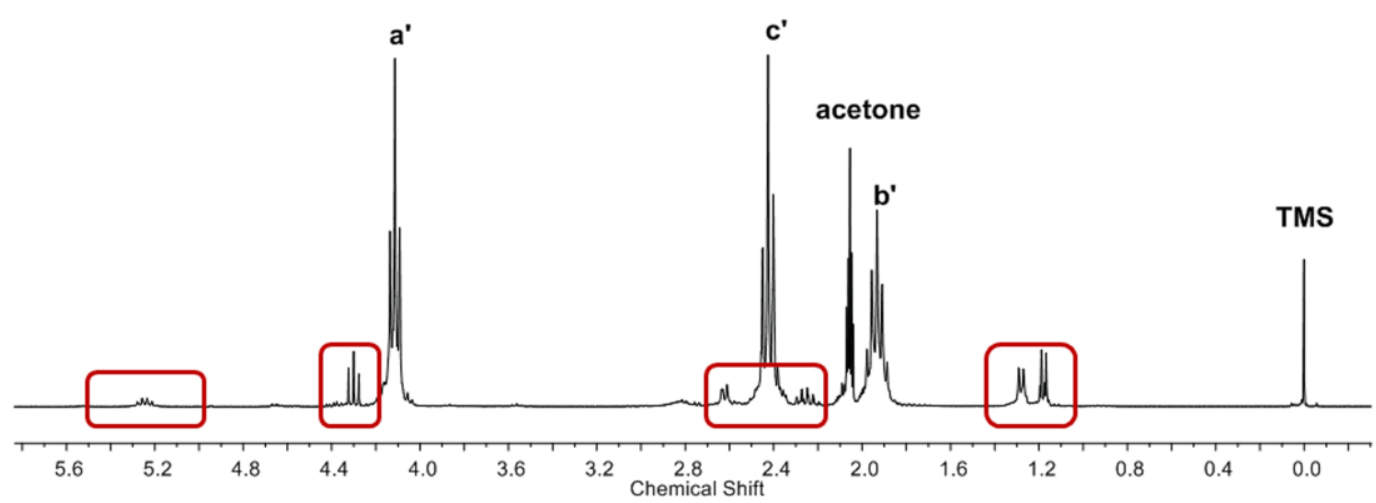

Since the DIF-polymer ICs have similar channel structures, there should be a reasonable ratio between the number of DIF molecules and the chain length of the incorporated guest polymers. Assuming the guest polymers all adopt an extended planar zigzag conformation as in their known polymorphs $^{9-11}$, the chain lengths of the guest polymers corresponding to one DIF molecule were calculated for DIF-PTHF, DIF-PCL and DIF-PBA ICs and listed in the following table. The chain lengths corresponding to one DIF molecule in the three ICs are close to each other, validating the rationality of the stoichiometric ratio determined by ${ }^{1} \mathrm{H}-\mathrm{NMR}$, even though the accurate conformations of the included polymers have not been determined yet.

\begin{tabular}{c|ccc}
\hline & DIF-PTHF & DIF-PCL & DIF-PBA \\
\hline $\begin{array}{c}\text { chain length } \\
\text { corresponding to one } \\
\text { DIF molecule }\end{array}$ & $2.41 \AA$ & $2.16 \AA$ & $2.37 \AA$ \\
\hline
\end{tabular}


Table S1. Crystallographic date for DIF-polymer ICs ${ }^{a}$ and the isostructural DIF solvates.

\begin{tabular}{|c|c|c|c|c|c|}
\hline & DIF-PTHF & DIF-PCL & DIF-chloroform ${ }^{c}$ & DIF-hexane ${ }^{d}$ & DIF-water ${ }^{e}$ \\
\hline empirical formula & $\mathrm{C}_{13} \mathrm{H}_{8} \mathrm{~F}_{2} \mathrm{O}_{3} \cdot 0.4 \mathrm{C}_{4} \mathrm{H}_{8} \mathrm{O}^{b}$ & $\mathrm{C}_{13} \mathrm{H}_{8} \mathrm{~F}_{2} \mathrm{O}_{3} \cdot 0.25 \mathrm{C}_{6} \mathrm{H}_{10} \mathrm{O}_{2}^{b}$ & $\mathrm{C}_{13} \mathrm{H}_{8} \mathrm{~F}_{2} \mathrm{O}_{3} \cdot 0.5 \mathrm{CHCl}_{3}$ & $\mathrm{C}_{13} \mathrm{H}_{8} \mathrm{~F}_{2} \mathrm{O}_{3} \cdot 0.25 \mathrm{C}_{6} \mathrm{H}_{14}$ & $\mathrm{C}_{13} \mathrm{H}_{8} \mathrm{~F}_{2} \mathrm{O}_{3} \cdot \mathrm{H}_{2} \mathrm{O}$ \\
\hline$M_{r}$ & 279.03 & 278.73 & 291.65 & 267.21 & 268.21 \\
\hline temperature (K) & $100(2)$ & $100(2)$ & $153(2)$ & $298(2)$ & $298(2)$ \\
\hline radiation type & Mo Ka & Mo Ka & Mo Ka & Mo Ka & Mo Ka \\
\hline crystal system & monoclinic & monoclinic & monoclinic & monoclinic & monoclinic \\
\hline space group & $C 2 / c$ & $P 2_{1} / n$ & $C 2 / c$ & $C 2 / c$ & $C 2 / c$ \\
\hline$a(\AA)$ & $34.411(18)$ & $9.907(4)$ & $34.2819(18)$ & $34.826(17)$ & $34.650(2)$ \\
\hline$b(\AA)$ & $3.651(2)$ & $3.7510(15)$ & $3.6771(2)$ & $3.7296(16)$ & $3.730(2)$ \\
\hline$c(\AA)$ & $20.746(11)$ & $32.415(13)$ & $20.7361(13)$ & $20.703(10)$ & $20.760(2)$ \\
\hline$\beta(\circ)$ & $110.537(8)$ & $95.141(6)$ & $110.376(2)$ & $110.63(5)$ & $110.47(6)$ \\
\hline$V\left(\AA^{3}\right)$ & $2441(2)$ & 1199.7(8) & $2450.4(2)$ & $2517(2)$ & $2513.7(14)$ \\
\hline$z$ & 8 & 4 & 8 & 8 & 8 \\
\hline$\rho_{\text {cal }}\left(\mathrm{g} \cdot \mathrm{cm}^{-3}\right)$ & 1.519 & 1.543 & 1.581 & 1.410 & 1.417 \\
\hline$\mu\left(\mathrm{mm}^{-1}\right)$ & 0.127 & 0.130 & 0.339 & 0.12 & 0.12 \\
\hline$\theta$ range $\left({ }^{\circ}\right)$ & $2.033-28.431$ & $2.103-28.286$ & $f$ & $1.25-24.97$ & $1.25-25$ \\
\hline index ranges & $\begin{array}{l}-46 \leq h \leq 36,-3 \leq k \leq 4 \\
-24 \leq 1 \leq 27\end{array}$ & $\begin{array}{l}-12 \leq \mathrm{h} \leq 13,-4 \leq \mathrm{k} \leq 3 \\
-41 \leq \mathrm{I} \leq 43\end{array}$ & $f$ & $\begin{array}{l}-40 \leq h \leq 38,-1 \leq k \leq 4 \\
-1 \leq 1 \leq 24\end{array}$ & $\begin{array}{l}-1 \leq h \leq 40,0 \leq k \leq 4, \\
-24 \leq \mathrm{I} \leq 23\end{array}$ \\
\hline collected/unique reflections & $7802 / 3051$ & $7665 / 2940$ & $f / 2162$ & 2312/2220 & $2306 / 2221$ \\
\hline data/restrains/parameters & $3051 / 0 / 168$ & 2940/0/168 & $f$ & 2220/18/227 & $2221 / 12 / 225$ \\
\hline$R_{\text {int }}$ & 0.0313 & 0.0296 & 0.1654 & 0.045 & 0.014 \\
\hline$R_{1}, w R_{2}(I>2 \sigma(I))$ & $0.0509,0.1353$ & $0.0430,0.1175$ & $0.0642,0.1449$ & $0.066,0.200$ & $0.050,0.178$ \\
\hline$R_{1}, w R_{2}$ (all data) & $0.0699,0.1470$ & $0.0564,0.1268$ & $f$ & $f$ & $f$ \\
\hline$S$ & 1.048 & 1.041 & 1.045 & 1.04 & 0.83 \\
\hline$\Delta \rho_{\max }, \Delta \rho_{\min }\left(\mathrm{e} \cdot \AA^{-3}\right)$ & $0.473,-0.531$ & $0.348,-0.299$ & $g$ & $0.27,-0.27$ & $-0.16,0.26$ \\
\hline
\end{tabular}

${ }^{a}$ The disordered guest polymers in DIF-polymer ICs were removed from the structural models using PLANTO/SQUEEZE ${ }^{4}{ }^{b}$ The stoichiometric ratios of DIF to monomers in DIF-polymer ICs were determined by ${ }^{1} \mathrm{H}$-NMR. ${ }^{c}$ Ref 1 (b). ${ }^{d}$ Ref $5 .{ }^{e}$ Ref $6 .{ }^{f}$ Original data not found. 
Table S2. Hydrogen-bonding geometry for DIF molecules in DIF-polymer ICs and the solvates.

\begin{tabular}{|c|c|c|c|c|}
\hline$D-\mathrm{H} \cdots A$ & $D-\mathrm{H}(\AA)$ & $H \cdots A(\AA)$ & $D \cdots A(\AA)$ & $D-\mathrm{H} \cdots A\left({ }^{\circ}\right)$ \\
\hline \multicolumn{5}{|l|}{ DIF-PTHF ${ }^{a}$} \\
\hline $\mathrm{O} 2-\mathrm{H} 2 \mathrm{O} \cdots \mathrm{O}^{i}$ & 0.840 & $1.797(1)$ & $2.637(2)$ & $177.6(1)$ \\
\hline $\mathrm{O} 3-\mathrm{H} 3 \mathrm{O} \cdots \mathrm{O} 1$ & 0.840 & $1.881(2)$ & $2.613(2)$ & $144.8(1)$ \\
\hline \multicolumn{5}{|l|}{ DIF-PCL ${ }^{a}$} \\
\hline $\mathrm{O} 2-\mathrm{H} 2 \mathrm{O} \cdots \mathrm{O} 1^{\mathrm{ii}}$ & 0.840 & $1.815(1)$ & 2.654(2) & 177.1(1) \\
\hline $\mathrm{O} 3-\mathrm{H} 3 \mathrm{O} \cdots \mathrm{O} 1$ & 0.840 & $1.908(1)$ & $2.634(2)$ & 143.9(1) \\
\hline \multicolumn{5}{|l|}{ DIF-chloroform ${ }^{b}$} \\
\hline $\mathrm{O} 2-\mathrm{H} 2 \mathrm{O} \cdots \mathrm{O}^{\mathrm{i}}$ & $0.81(4)$ & $1.83(4)$ & $2.636(3)$ & $177(5)$ \\
\hline $\mathrm{O} 3-\mathrm{H} 3 \mathrm{O} \cdots \mathrm{O} 1$ & $0.90(6)$ & $1.82(7)$ & $2.607(4)$ & $145(6)$ \\
\hline \multicolumn{5}{|l|}{ DIF-hexane $^{c}$} \\
\hline $\mathrm{O} 2-\mathrm{H} 2 \mathrm{O} \cdots \mathrm{O} 1^{\mathrm{i}}$ & $1.02(5)$ & $1.69(5)$ & $2.665(6)$ & $159(3)$ \\
\hline $\mathrm{O}-\mathrm{H} 3 \mathrm{O} \cdots \mathrm{O} 1$ & $0.92(5)$ & $2.01(6)$ & $2.630(6)$ & $123(3)$ \\
\hline \multicolumn{5}{|l|}{ DIF-water ${ }^{d}$} \\
\hline $\mathrm{O} 2-\mathrm{H} 2 \mathrm{O} \cdots \mathrm{O}^{\mathrm{i}}$ & $1.00(4)$ & $1.65(4)$ & $2.650(3)$ & $176(4)$ \\
\hline $\mathrm{O}-\mathrm{H} 3 \mathrm{O} \cdots \mathrm{O} 1$ & $1.11(6)$ & $1.75(6)$ & $2.620(4)$ & $131(4)$ \\
\hline
\end{tabular}

symmetry codes: (i) 1/2-x, 1/2-y, 1-z; (ii) 1-x, -y, -z.

${ }^{a}$ This work. ${ }^{b} \operatorname{Ref} 1$ (b). ${ }^{c} \operatorname{Ref} 5 .{ }^{d} \operatorname{Ref} 6$.

Table S3. $\pi-\pi$ stacking geometry for DIF molecules in DIF-polymer ICs and the solvates.

\begin{tabular}{cccccc}
\hline & DIF-PTHF $^{d}$ & DIF-PCL $^{d}$ & DIF-chloroform $^{e}$ & DIF-hexane $^{f}$ & DIF-water $^{g}$ \\
\hline$\angle\left(\mathrm{Ph}_{\mathrm{A}}, \mathrm{Ph}_{\mathrm{B}}\right)(\circ)^{a}$ & $43.1(1)$ & $36.0(1)$ & $43.2(1)$ & $43.2(2)$ & $43.2(1)$ \\
$R_{\mathrm{d}, \mathrm{A}}(\AA)^{b}$ & $3.405(1)$ & $3.502(1)$ & $3.432(2)$ & $3.477(2)$ & $3.479(2)$ \\
$R_{\mathrm{d}, \mathrm{B}}(\AA)^{b}$ & $3.383(1)$ & $3.375(1)$ & $3.402(1)$ & $3.452(5)$ & $3.452(2)$ \\
$R_{\text {shift, }}(\AA)^{c}$ & 1.32 & 1.34 & 1.32 & 1.35 & 1.34 \\
$R_{\text {shitt, }}(\AA)^{c}$ & 1.37 & 1.64 & 1.40 & 1.41 & 1.41
\end{tabular}

${ }^{a}$ Dihedral angle formed by phenyl ring $A$ and B. ${ }^{b}$ Distance between the neigbouring parallel phenyl rings. ${ }^{c}$ Shift distance of centroids between the neighbouring parallel phenyl rings. ${ }^{d}$ This work. ${ }^{e} \operatorname{Ref} 1$ (b). ${ }^{f} \operatorname{Ref} 5 .{ }^{g} \operatorname{Ref} 6$.

Table S4. Selected torsion angles characterizing the conformation of DIF molecules in DIF-polymer ICs and the solvates.

\begin{tabular}{lccccc}
\hline torsion angle (०) & DIF-PTHF $^{a}$ & DIF-PCL $^{a}$ & DIF-chloroform $^{b}$ & DIF-hexane $^{c}$ & DIF-water $^{d}$ \\
\hline$\theta 1$ = C2-C3-O3-H3O & $1.6(2)$ & $2.6(2)$ & $6(4)$ & $7(4)$ & $4(4)$ \\
$\theta 2$ = O1-C1-O2-H2O & $0.6(2)$ & $4.1(2)$ & $0(3)$ & $12(3)$ & $2(2)$ \\
$\theta 3$ = O2-C1-C2-C7 & $0.7(2)$ & $0.0(2)$ & $0.2(4)$ & $0.3(7)$ & $0.8(4)$ \\
$\theta 4$ = C9-C8-C6-C7 & $42.8(2)$ & $35.9(2)$ & $43.3(4)$ & $43.4(8)$ & $43.2(4)$ \\
\hline
\end{tabular}

${ }^{a}$ This work. ${ }^{b}$ Ref $1(\mathrm{~b}) .{ }^{c}$ Ref $5 .{ }^{d}$ Ref 6. 


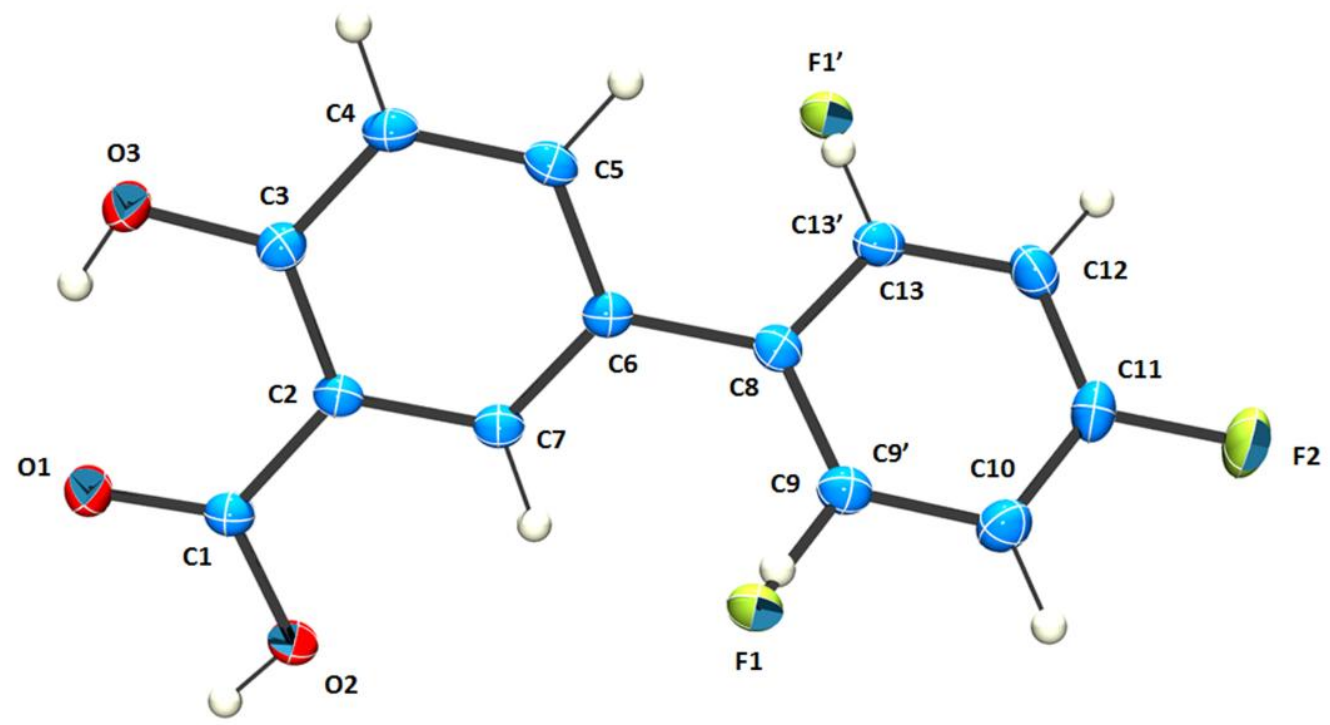

(a)

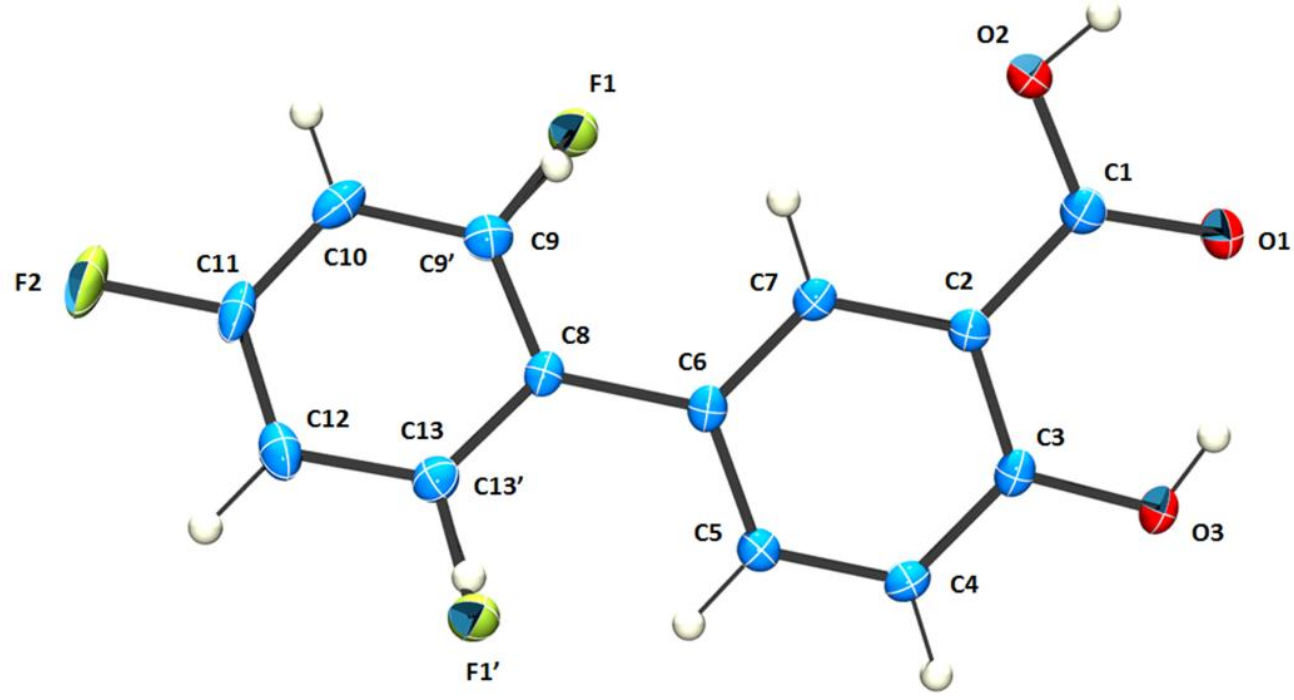

(b)

Figure S3. An ORTEP plot of DIF molecules in (a) DIF-PTHF IC and (b) DIF-PCL IC with the atomic numbering scheme. Displacement ellipsoids are drawn at the 50\% probability level. Notice that the fluorine atoms are disordered over the two ortho sites on the phenyl rings, each with a site occupancy factor of 0.5 . 


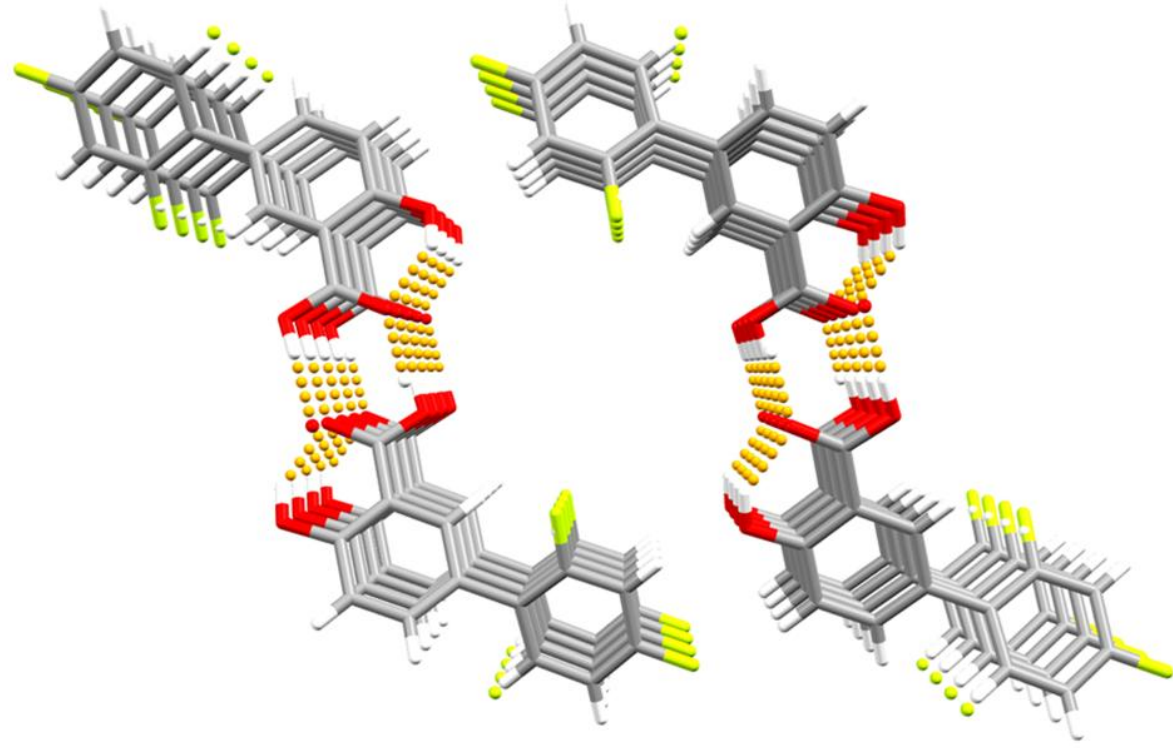

(a)

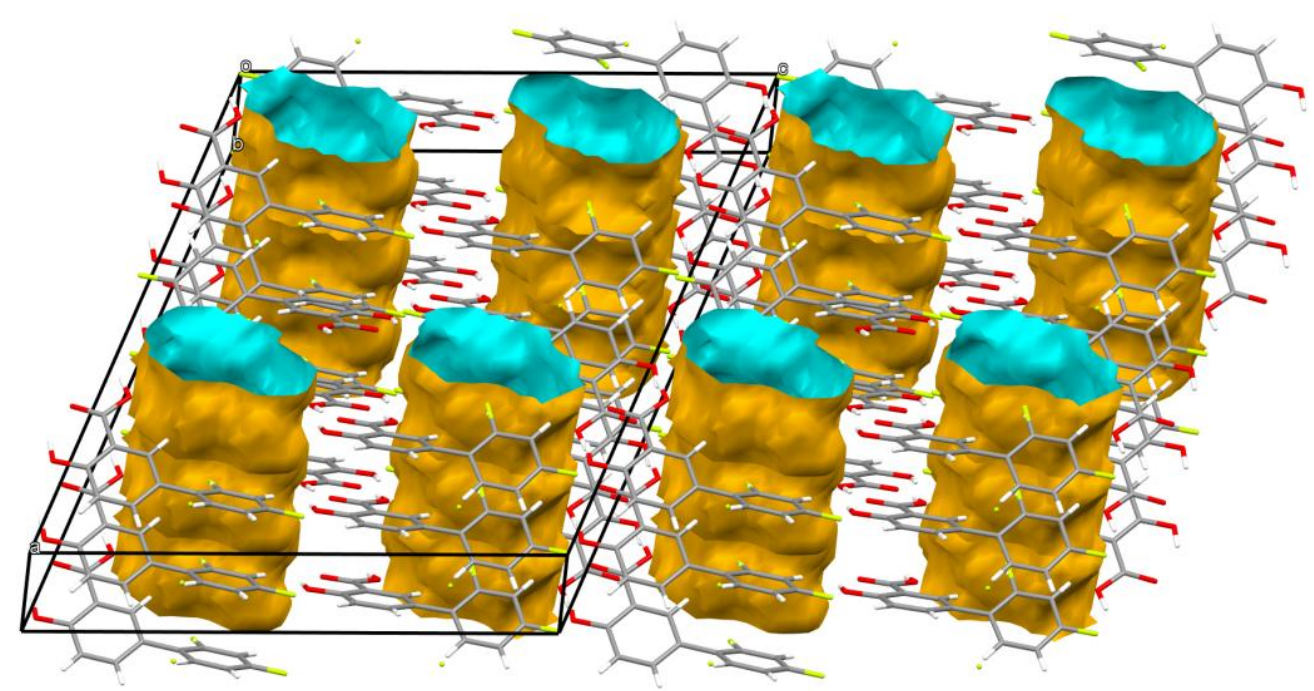

(b)

Figure S4. (a) Crystal structure packing patterns of DIF-PTHF IC with the guest molecules removed, viewed along the crystallographic b-axis. The structure exhibits hydrogen-bonded DIF dimers which form into stacks involving $\pi-\pi$ stacking interactions from the neighboring parallel molecules. The stacks are related to each other by phenyl $\mathrm{H} \cdots \mathrm{H}, \mathrm{H} \cdots \mathrm{F}$ and $\mathrm{H} \cdots \mathrm{O}$ contacts leaving continuous channels along the b-axis. (b) The channels within DIF-PTHF IC correspond to the space displayed using a probe radius of $1.2 \AA$ and a grid spacing of $0.5 \AA$. 


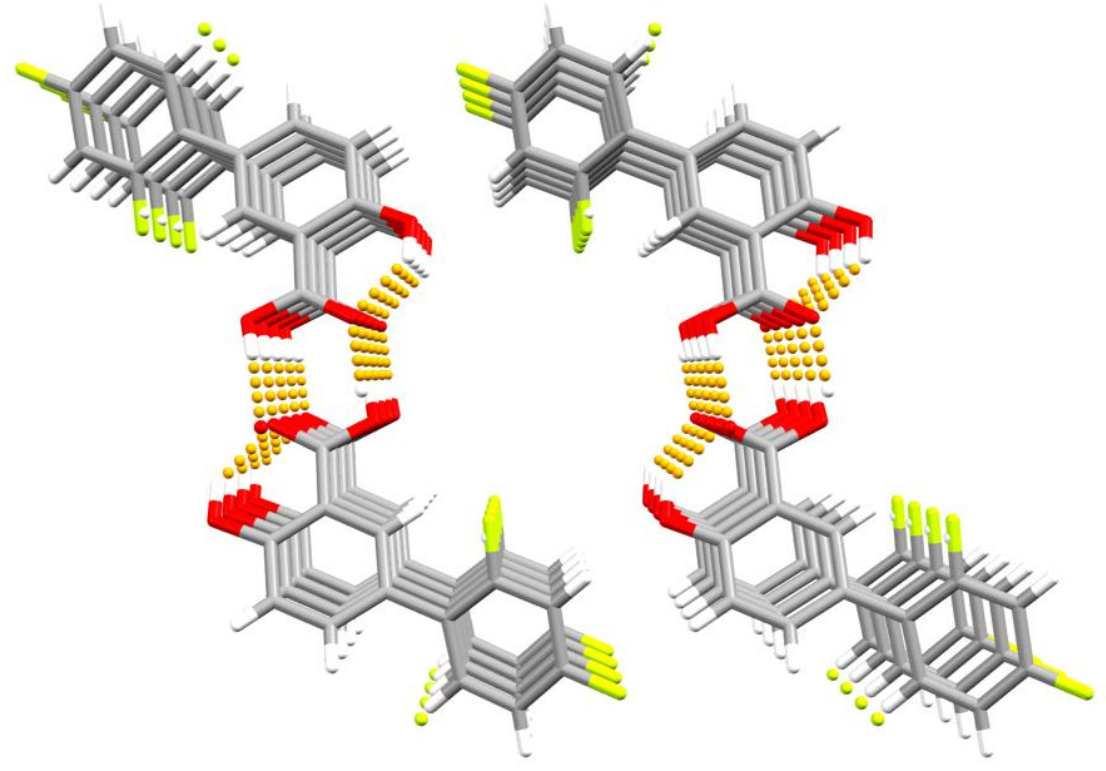

(a)

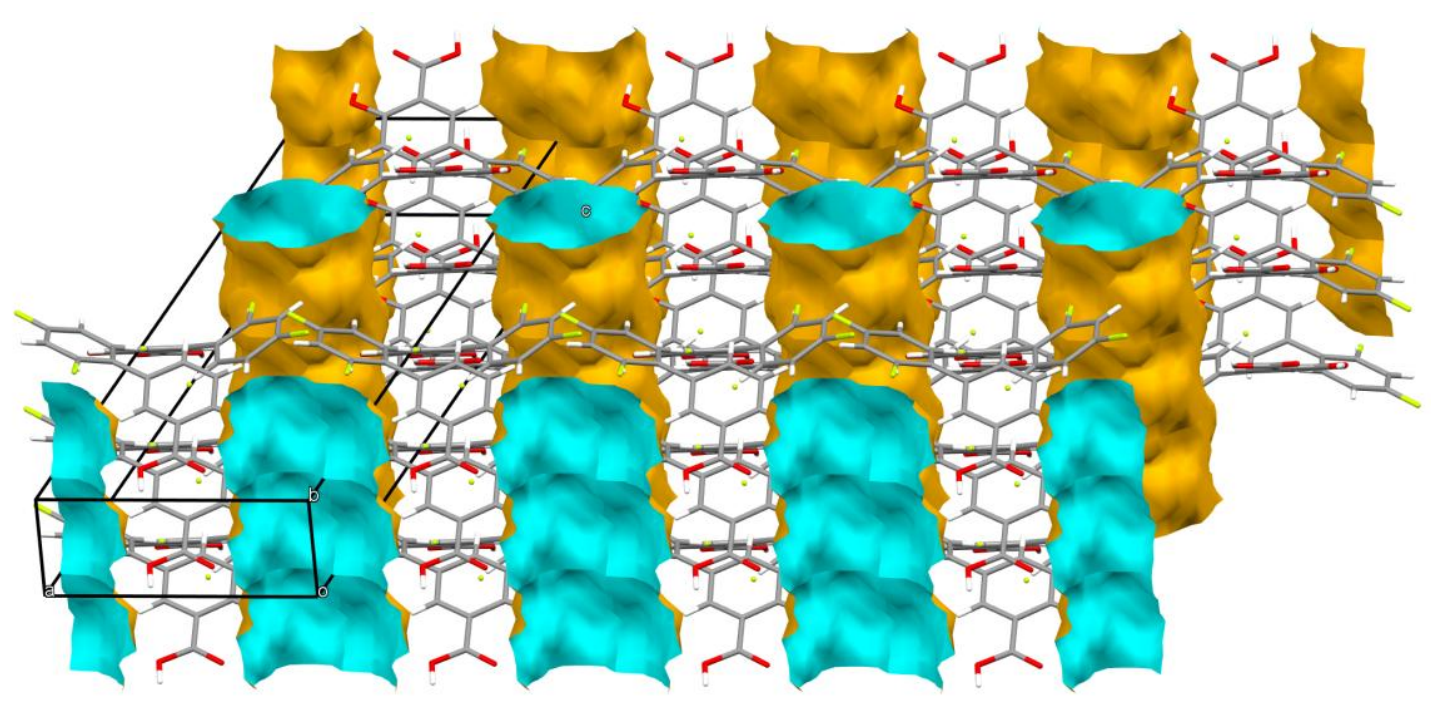

(b)

Figure S5. (a) Crystal structure packing patterns of DIF-PCL IC with the guest molecules removed, viewed along the crystallographic b-axis. The structure exhibits hydrogen-bonded DIF dimers which form into stacks involving $\pi-\pi$ stacking interactions from the neighboring parallel molecules. The stacks are related to each other by phenyl $\mathrm{H} \cdots \mathrm{H}, \mathrm{H} \cdots \mathrm{F}$ and $\mathrm{H} \cdots \mathrm{O}$ contacts leaving continuous channels along the b-axis. (b) The channels within DIF-PCL IC correspond to the space displayed using a probe radius of $1.2 \AA$ and a grid spacing of $0.5 \AA$. 

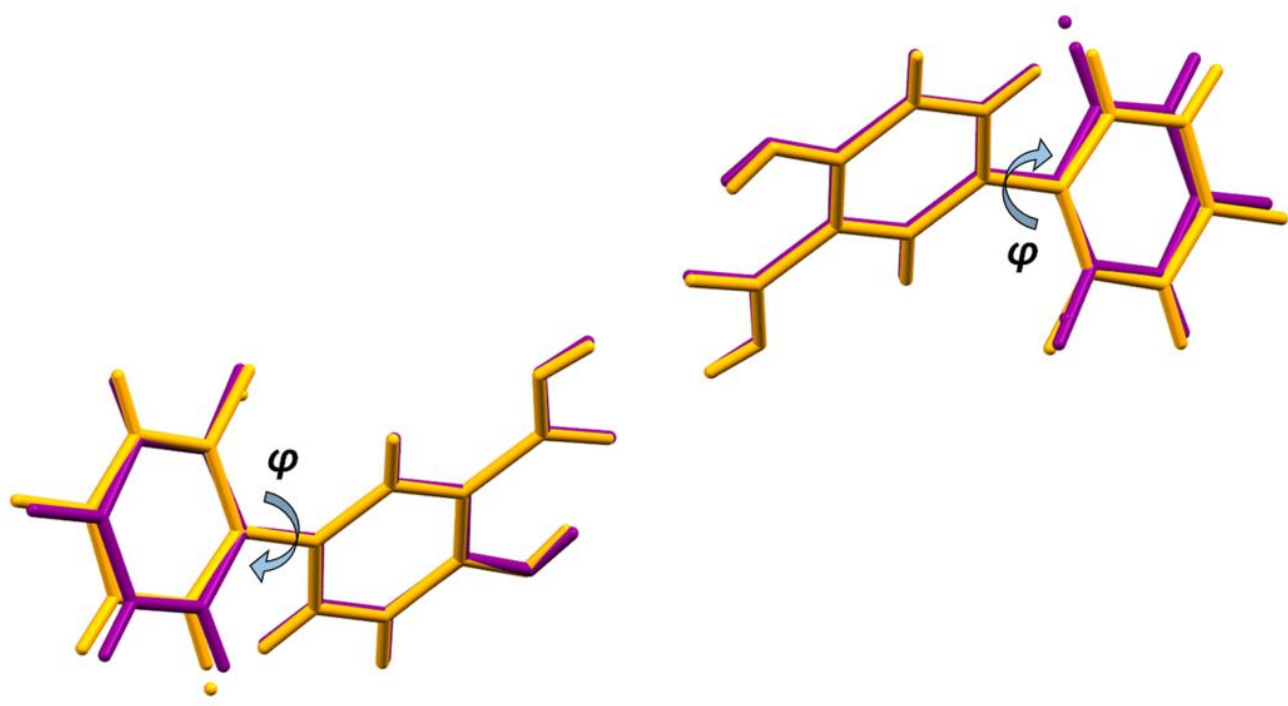

(a)

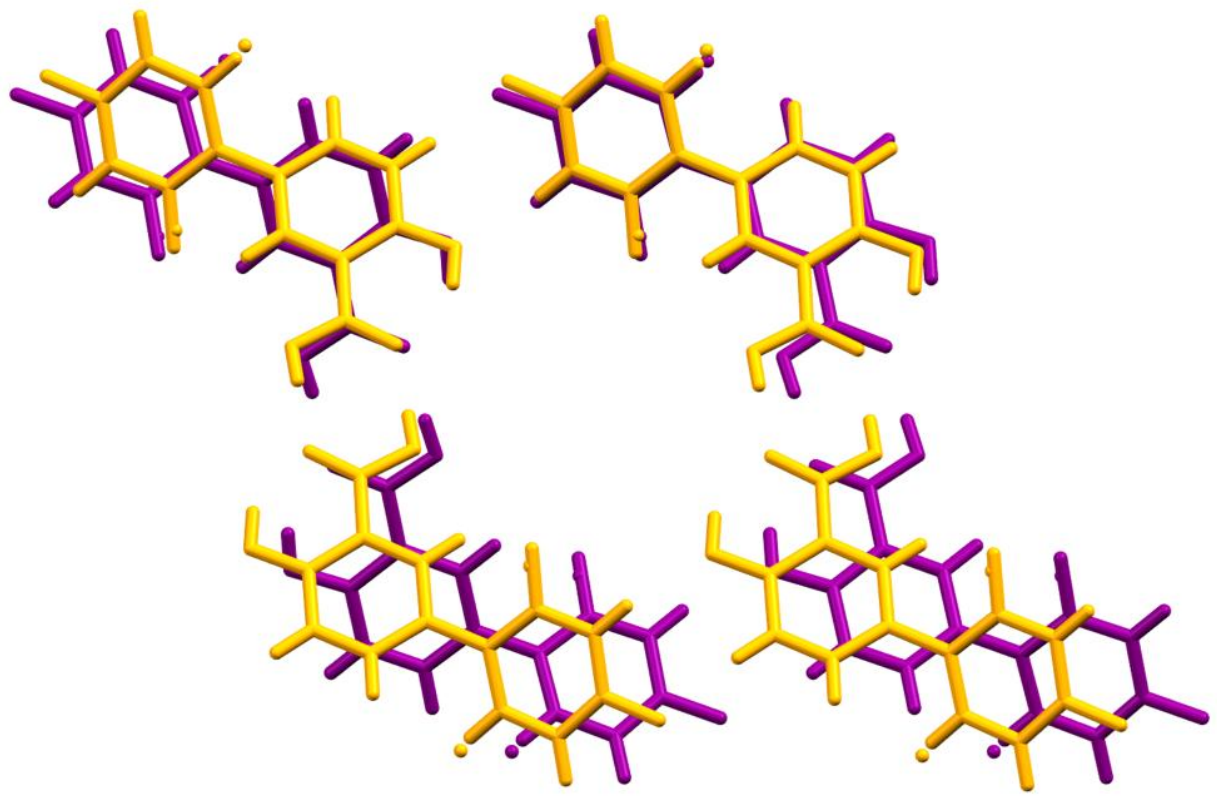

(b)

Figure S6. (a) Overlap of DIF dimers in DIF-PTHF (purple) and DIF-PCL (yellow) ICs. The difference in conformation of the two dimers mainly arises from the different torsion angles $(\varphi=$ $43.1^{\circ}$ and $36.0^{\circ}$, respectively) between the linked phenyl rings. (b) Overlap of the channel structures in DIF-PTHF (purple) and DIF-PCL (yellow) ICs. A smaller cross-sectional area of the channel can be observed for DIF-PCL compared with DIF-PTHF. 

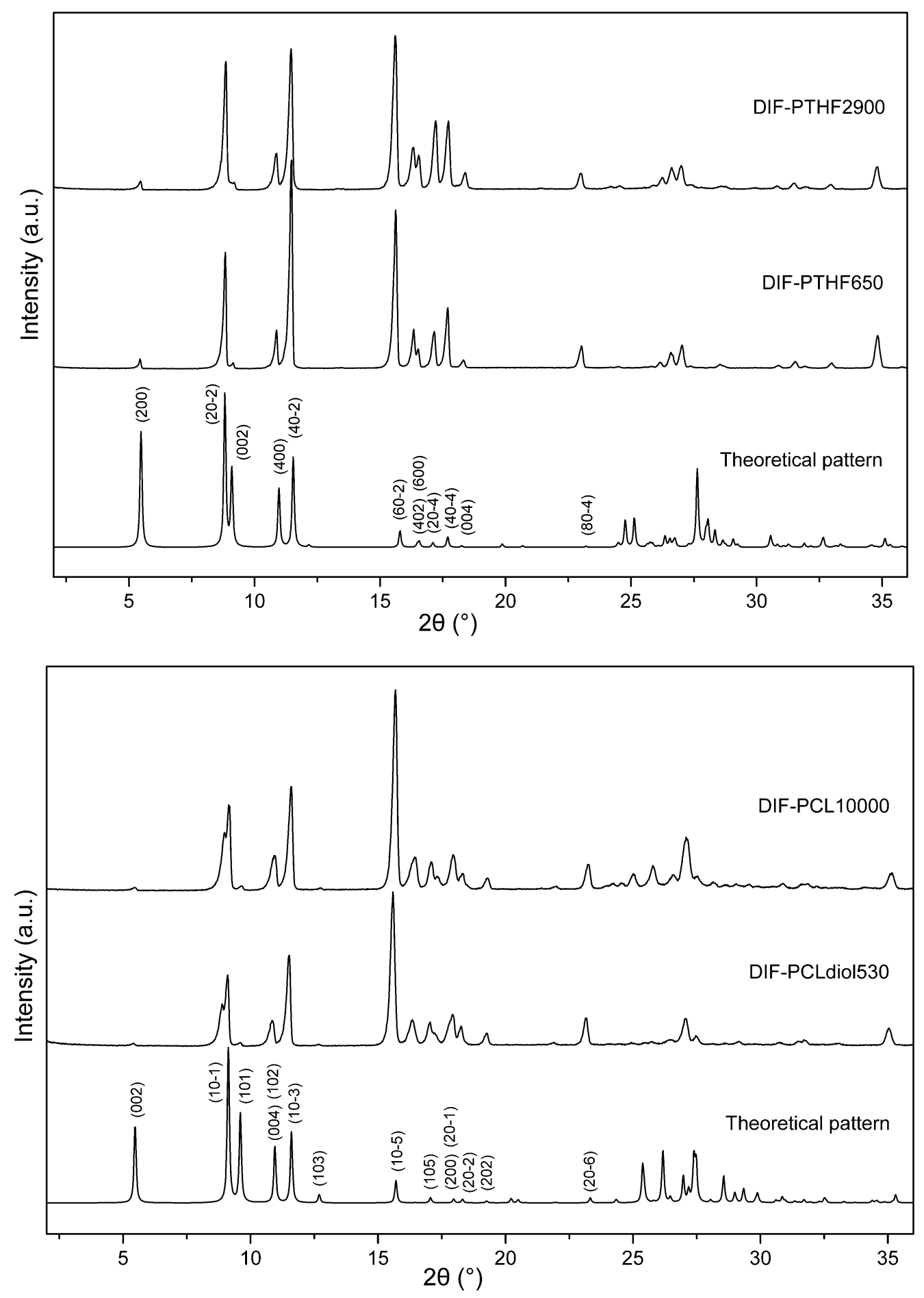

Figure S7. Comparison of the experimental and theoretical PXRD patterns for DIF-PTHF (top) and DIF-PCL (bottom) ICs. The theoretical patterns were calculated from single crystal data using Mercury $^{7}$ at a wavelength $1.54056 \AA$, and showed good agreement with the experimental patterns, validating the structural consistency between the single crystal and the powder crystal. Prominent $h k l$ planes are indicated. The small differences between experimental and theoretical patterns may arise from the guest polymers which cannot be modeled in the single-crystal structures. 


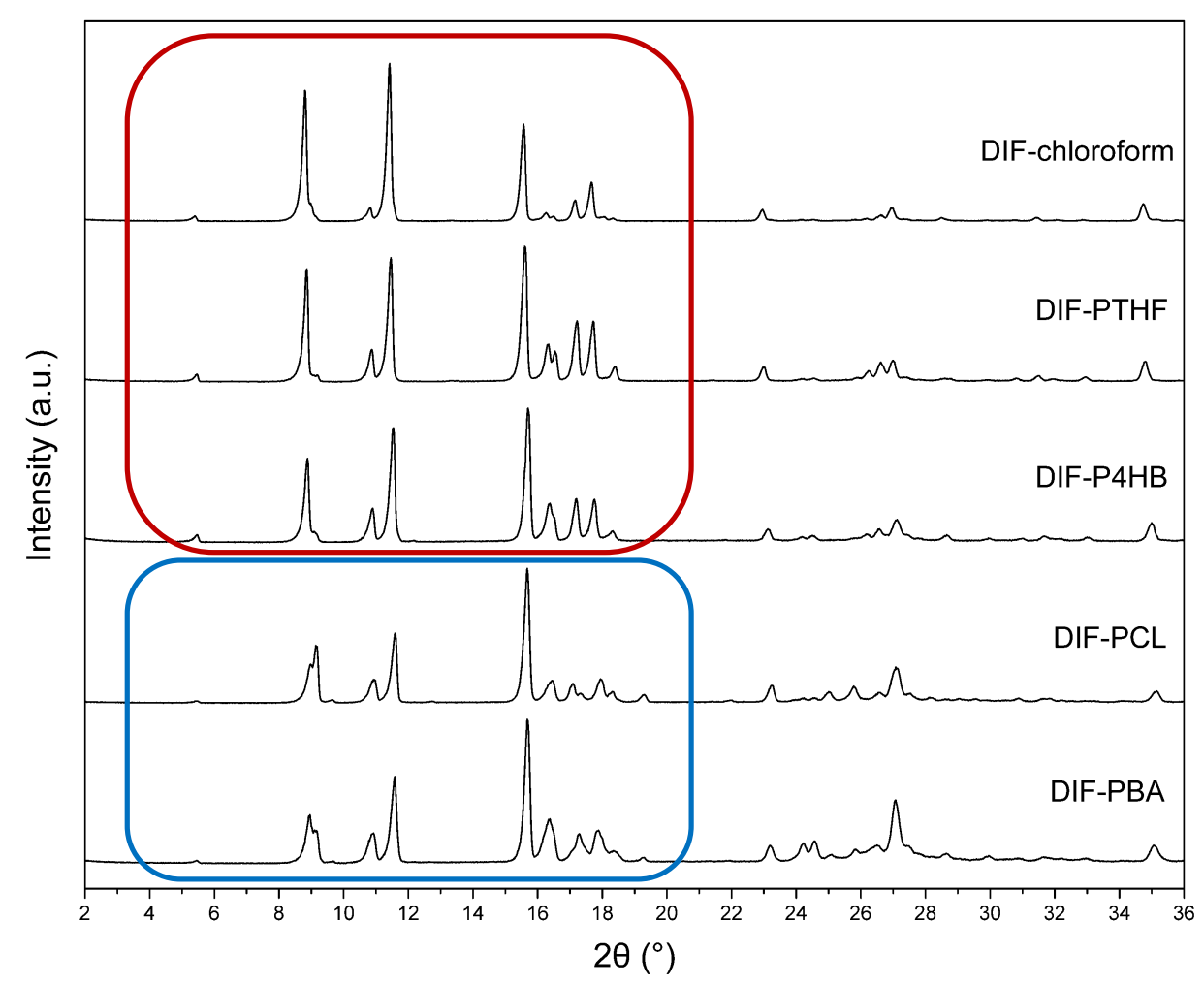

(a)

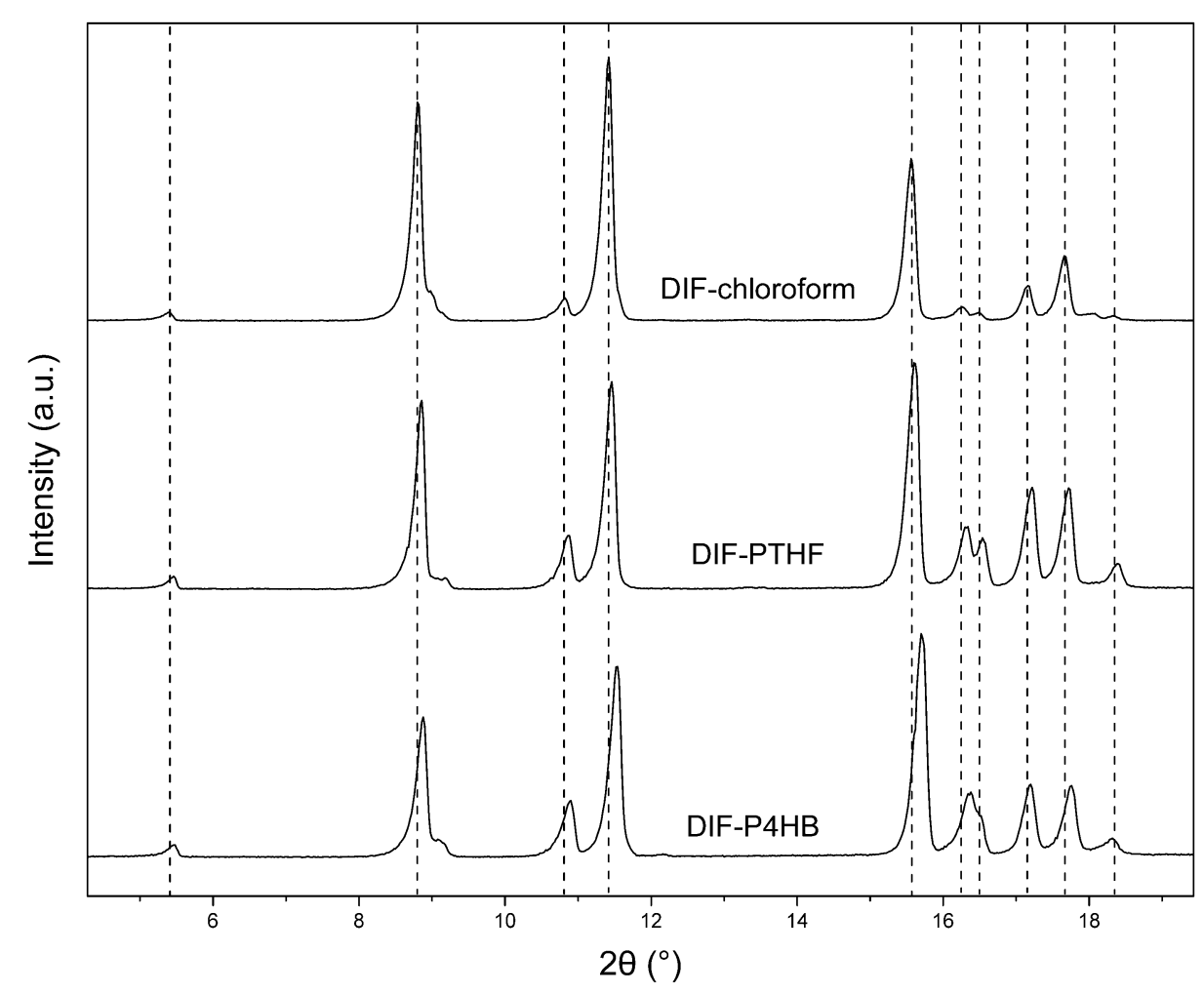

(b) 


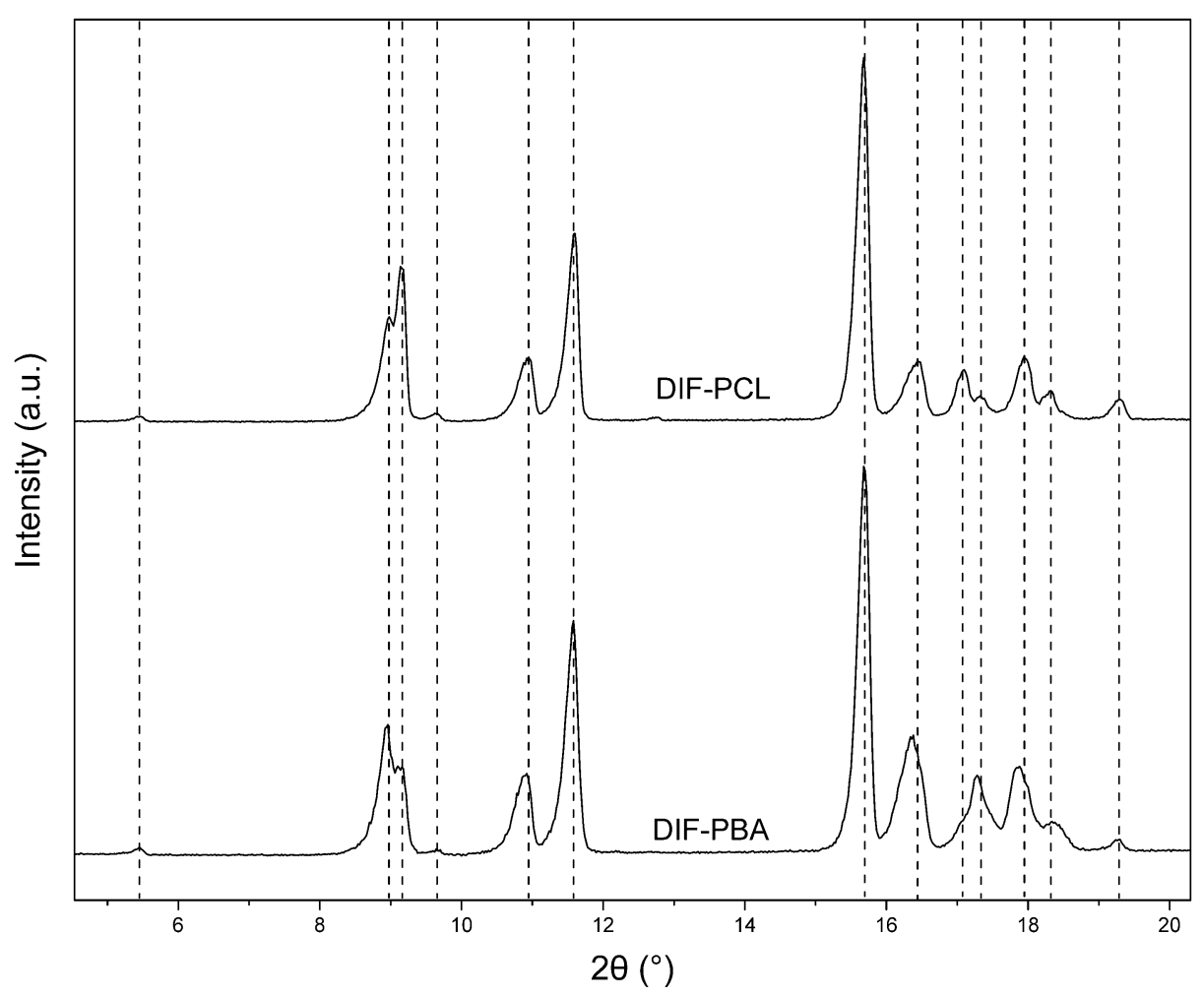

(c)

Figure S8. (a) Comparison of the PXRD patterns of DIF-chloroform solvate and DIF-polymer ICs. Two groups of similar reflection patterns can be distinguished, as shown by the red and blue wireframes respectively. (b) Diffractograms of DIF-chloroform, DIF-PTHF and DIF-P4HB showed almost the same characteristic peaks in the range of $5-20^{\circ} 2 \theta$ (the red wireframe in (a)), indicating that DIF-P4HB is likely to adopt a $C 2 / c$ framework similar to those in DIF-chloroform and DIF-PTHF. (c) DIF-PCL and DIF-PBA produced similar reflections and showed small differences from those in (b) especially in the range of $8-10^{\circ}$ and $16-20^{\circ} 2 \theta$, which suggest that DIF-PBA may have a $P 2_{1} / n$ framework as in DIF-PCL. In addition, slight changes in the peak position can still be observed for both sets of reflections when accommodating different guest molecules, suggesting the channel dimensions in DIF frameworks can also vary with the guest molecules. 


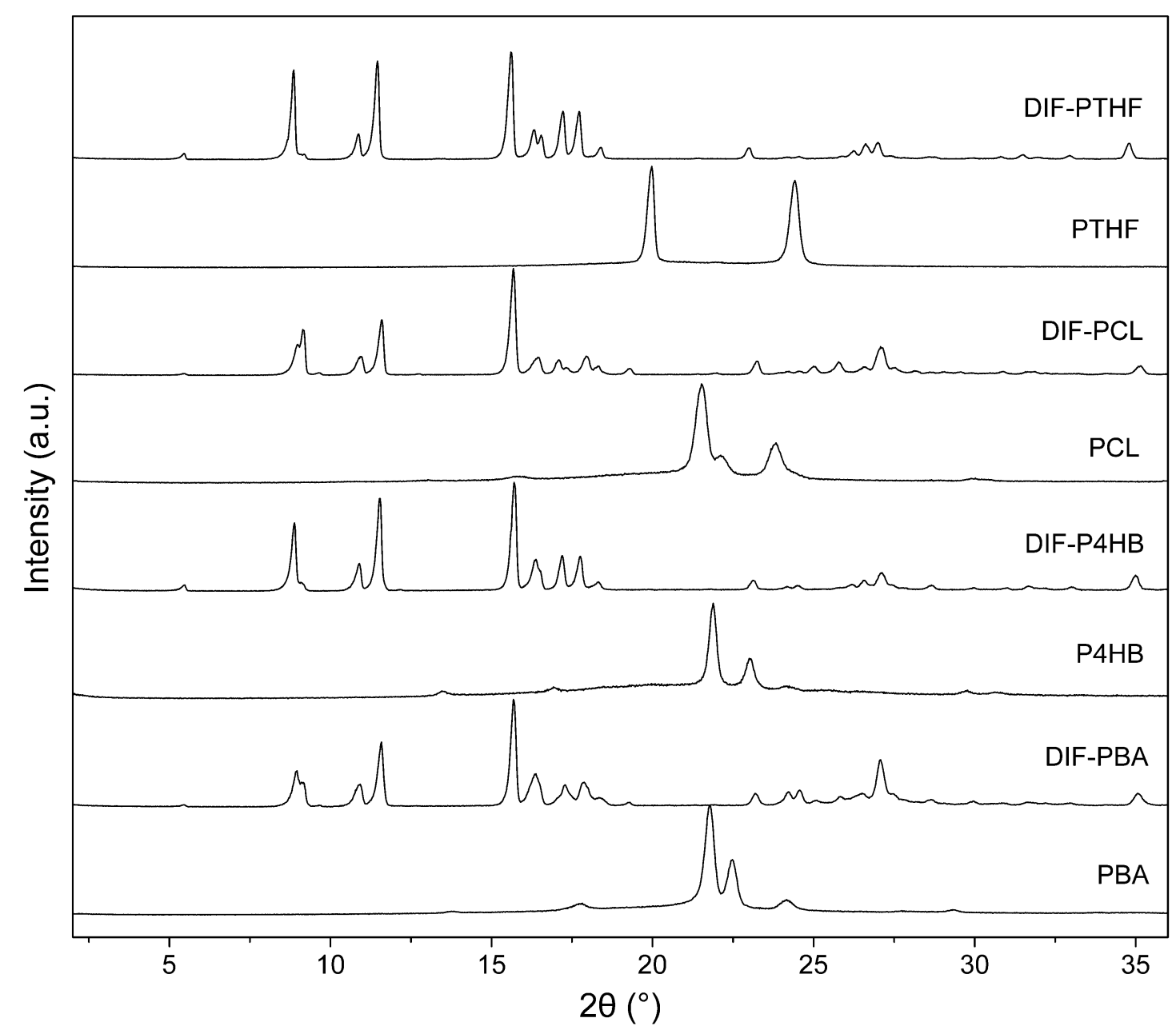

Figure S9. Comparison of the PXRD patterns of DIF-polymer ICs and the corresponding pure guest polymers. The absence of reflections characteristic of the guest polymers in the patterns of DIF-polymer ICs indicates the purity of these IC crystals. The peaks at $13.5^{\circ}$ and $16.9^{\circ} 2 \theta$ for P4HB sample may arise from the P3HB component in the raw material. 


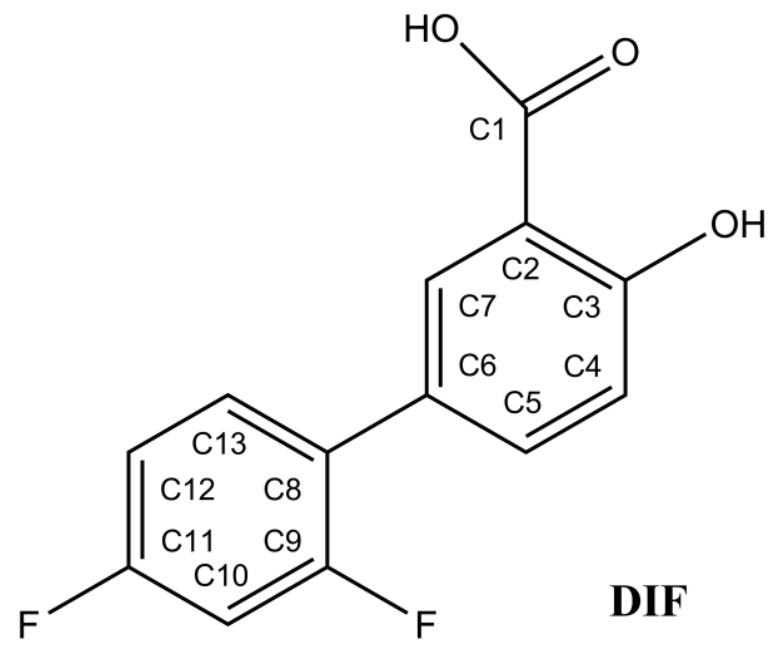

(a)

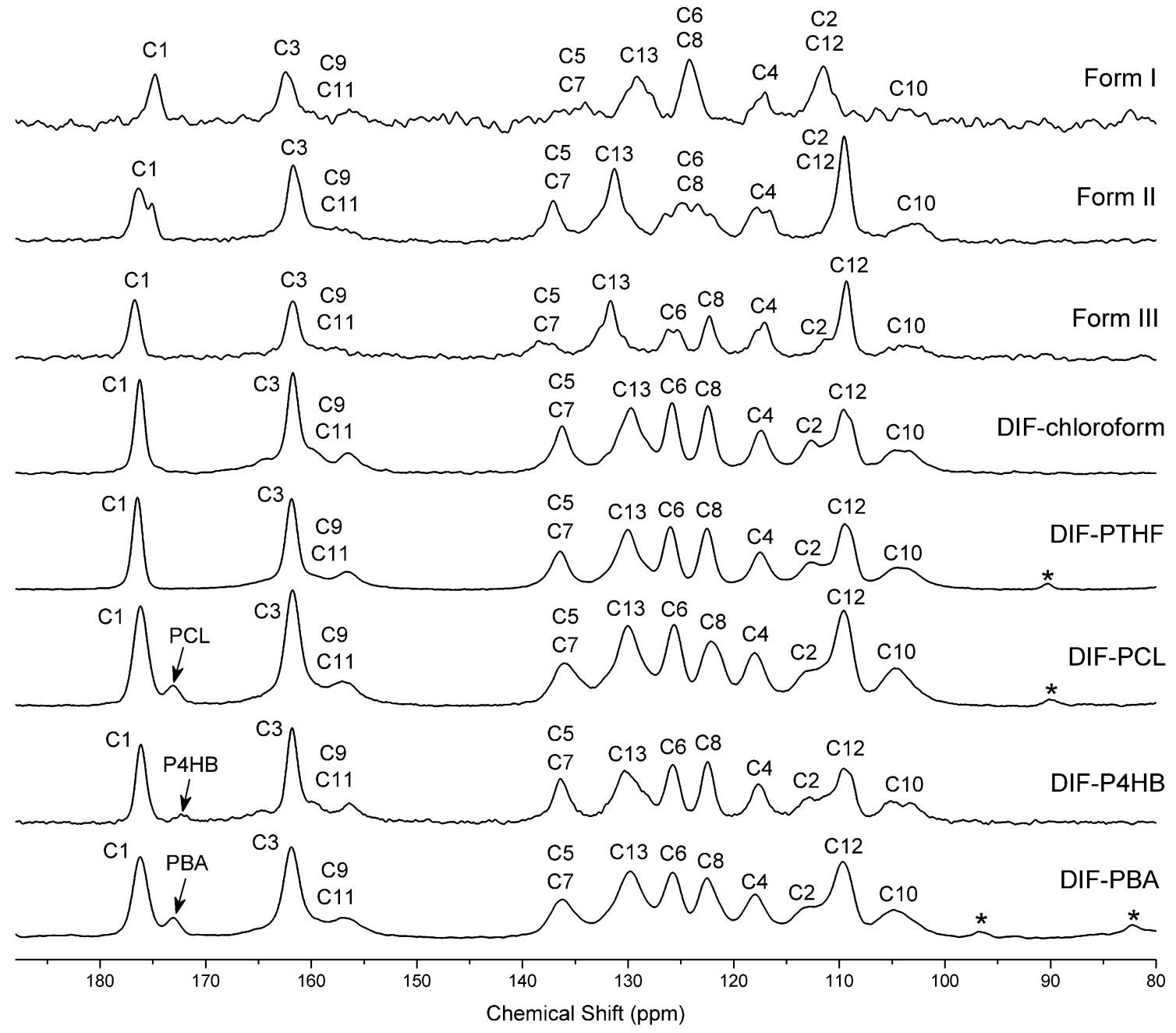

(b) 


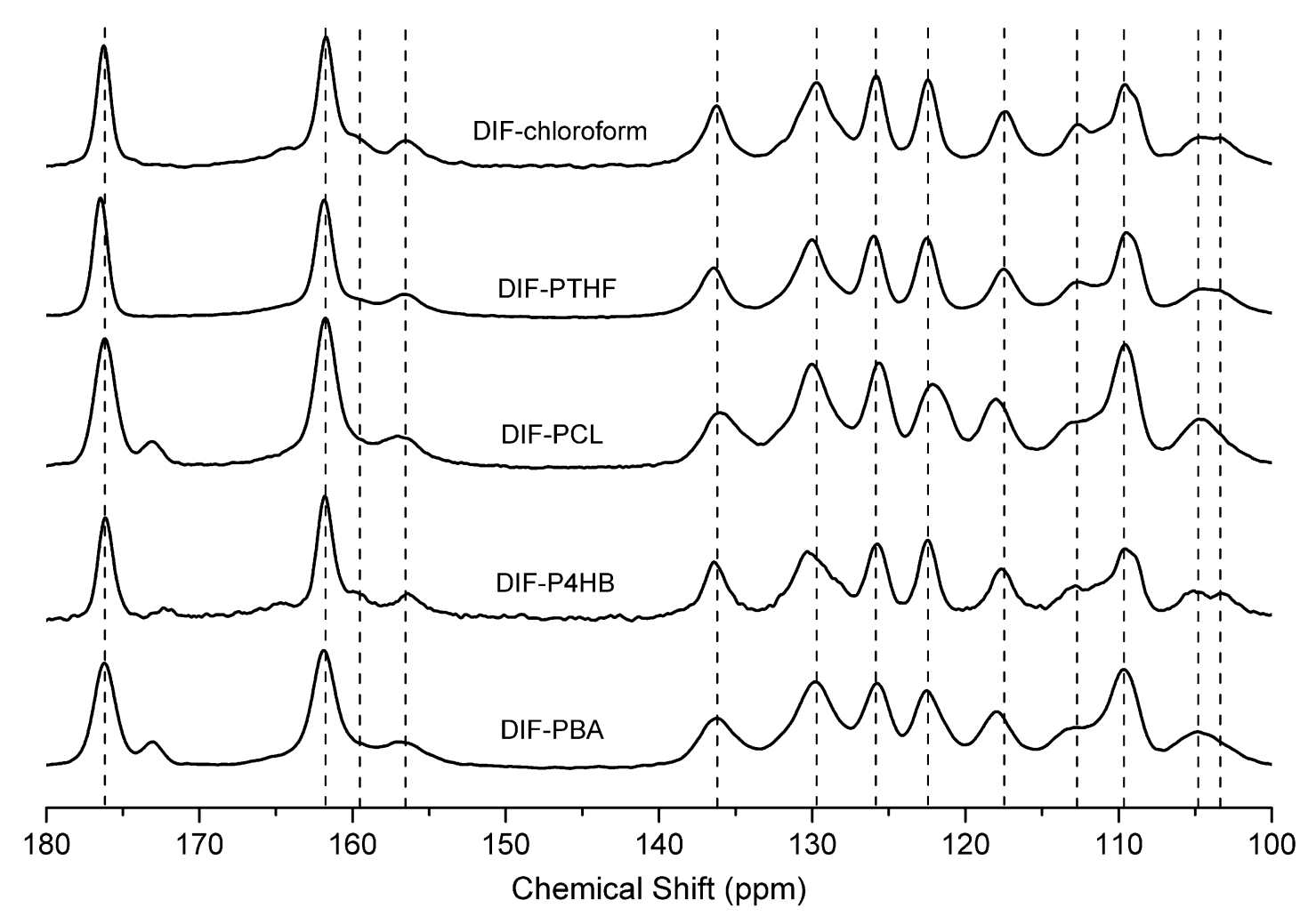

(C)

Figure S10. CP/MAS ${ }^{13} \mathrm{C}$ spectra of DIF pure forms, DIF-chloroform solvate and DIF-polymer ICs. Molecular structure of DIF with the atomic numbering scheme is shown in (a). A detailed comparison of the characteristic ${ }^{13} \mathrm{C}$ resonances for DIF molecules in the different crystalline forms is shown in (b). The peak assignment was performed in reference to the previously reported studies $^{8}$. All four DIF-polymer ICs produced ${ }^{13} \mathrm{C}$ resonances significantly different from those of the known DIF polymorphs, but showed close similarities to the chloroform solvate, which can be attributed to the similar DIF frameworks adopted by IC crystals and chloroform solvate. A detailed comparison of the spectra for chloroform solvate and IC crystals in the chemical shift range from 180-100 ppm is presented in (c). Small differences can be found in the ${ }^{13} \mathrm{C}$ peak position and splitting (e.g., for C10) characteristic of the DIF frameworks, which presumably arise from the changes in channel composition and channel structure caused by the inclusion of different guest molecules. The resonances marked with asterisks indicate spinning sidebands. 


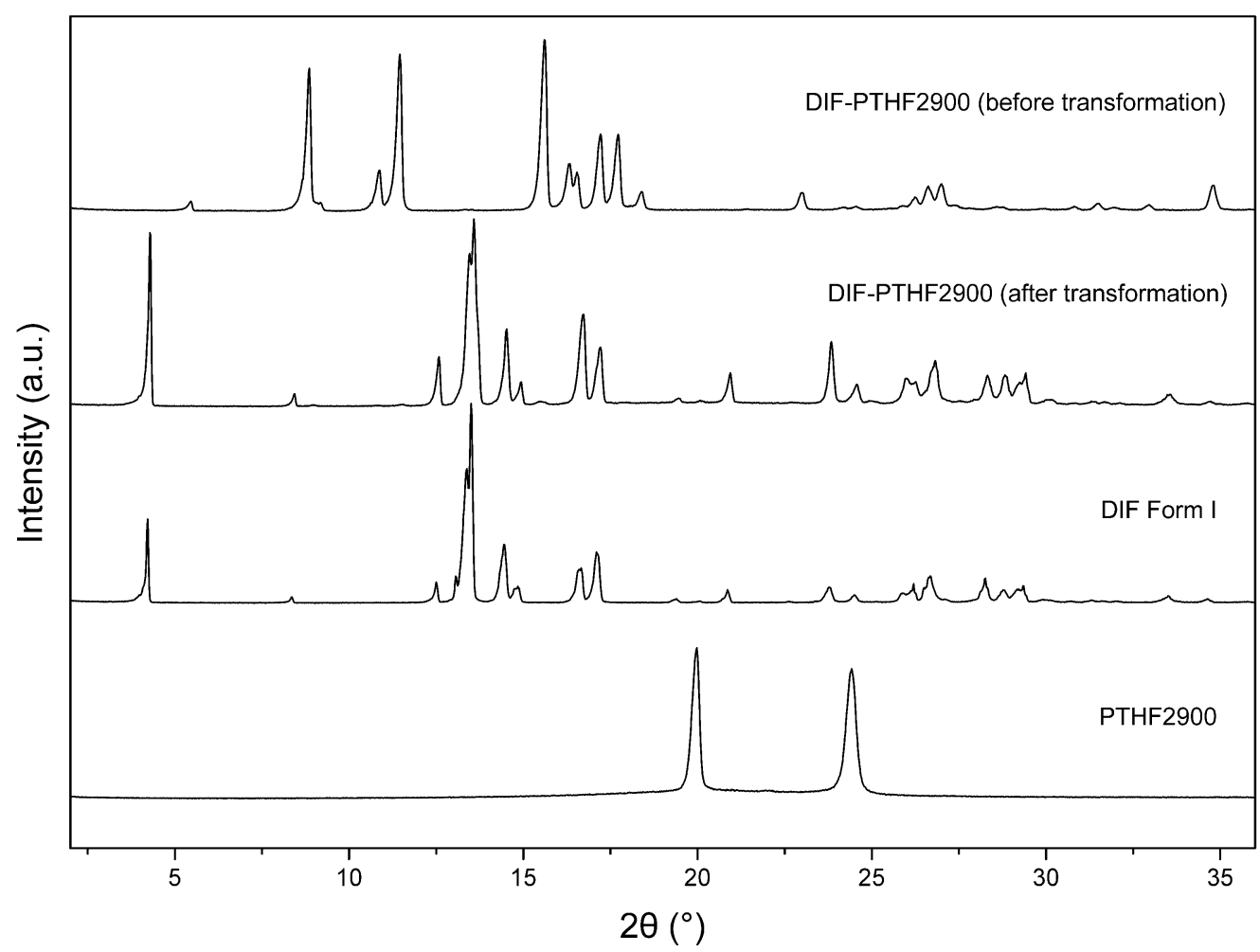

(a)

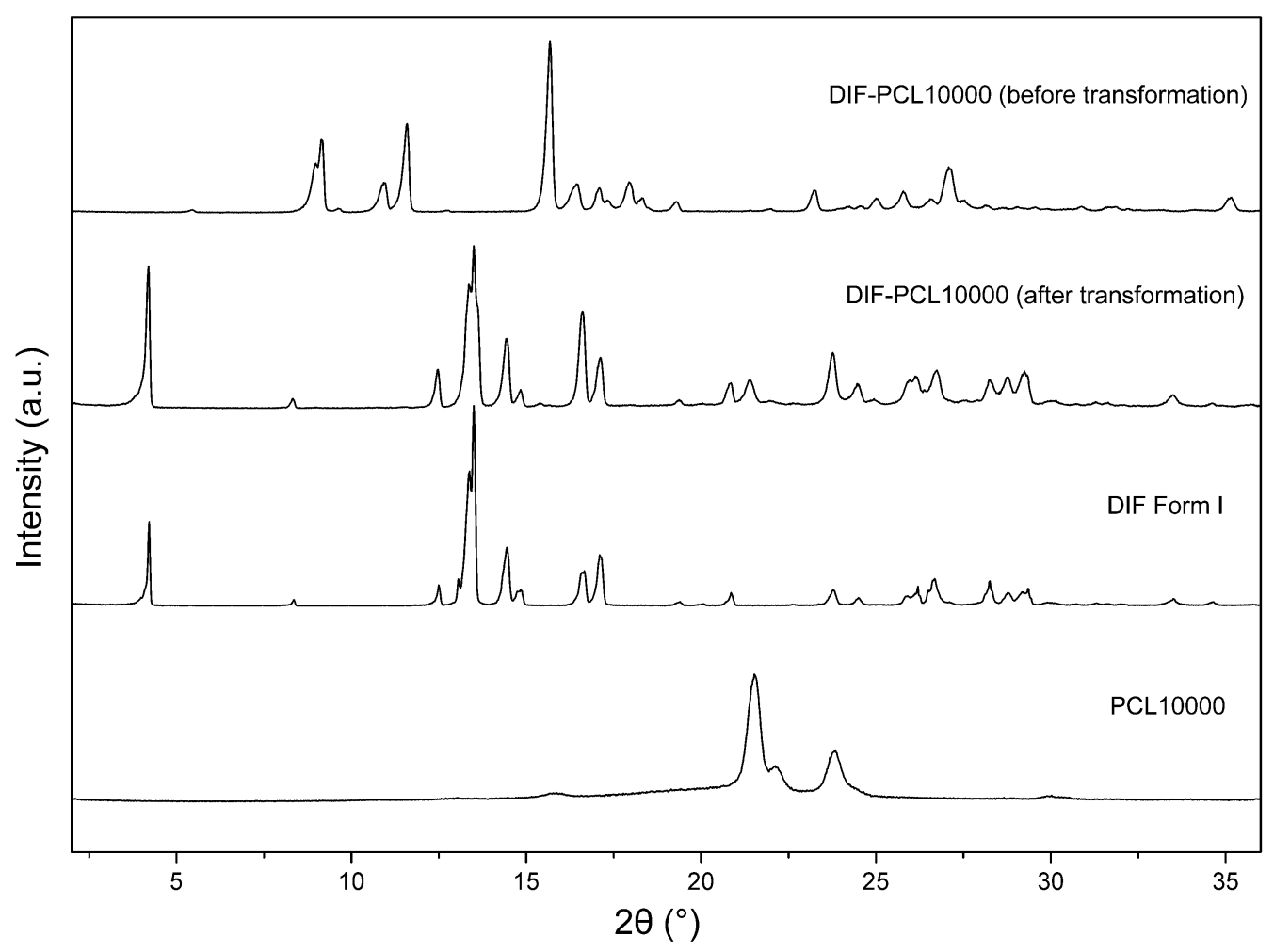

(b)

Figure S11. Comparison of the PXRD patterns before and after the thermal transformation of (a) DIF-PTHF and (b) DIF-PCL ICs. The characteristic peaks appeared after transformation indicate 
the formation of DIF Form I during the dissociation and recrystallization process. All the measurements were performed at room temperature. Peaks corresponding to PCL crystals can be observed in the transformed DIF-PCL sample, while characteristic peaks of PTHF crystals did not evidently appeared in the transformed DIF-PTHF sample probably due to its low crystallinity. 

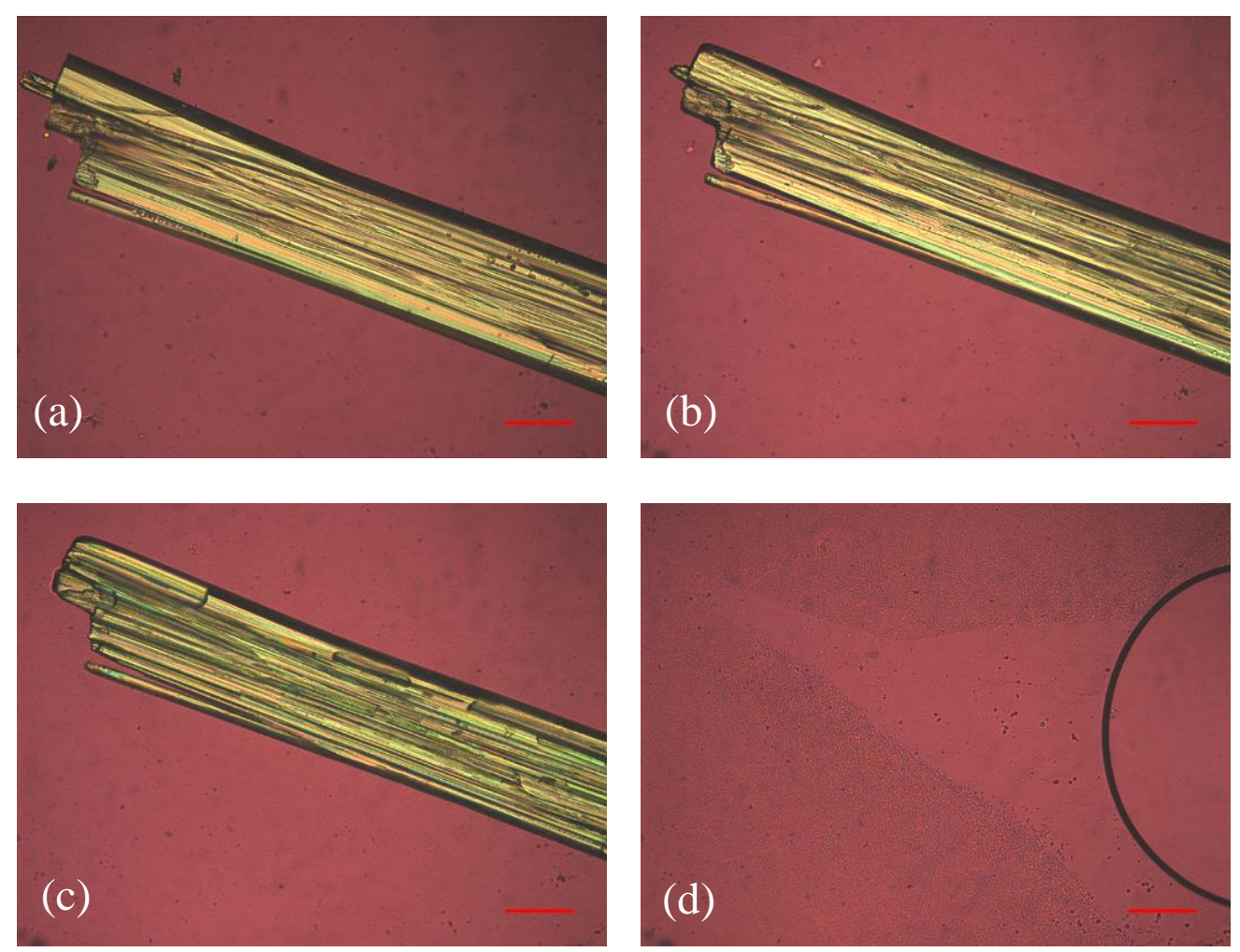

Figure S12. POM observation of the dissociation and recrystallization of DIF-PTHF650 IC crystal at a heating rate of $10{ }^{\circ} \mathrm{C} / \mathrm{min}$. (a) r.t., (b) $150{ }^{\circ} \mathrm{C}$, (c) $166^{\circ} \mathrm{C}$ and (d) $214{ }^{\circ} \mathrm{C}$. The bars in these pictures represent $100 \mu \mathrm{m}$.
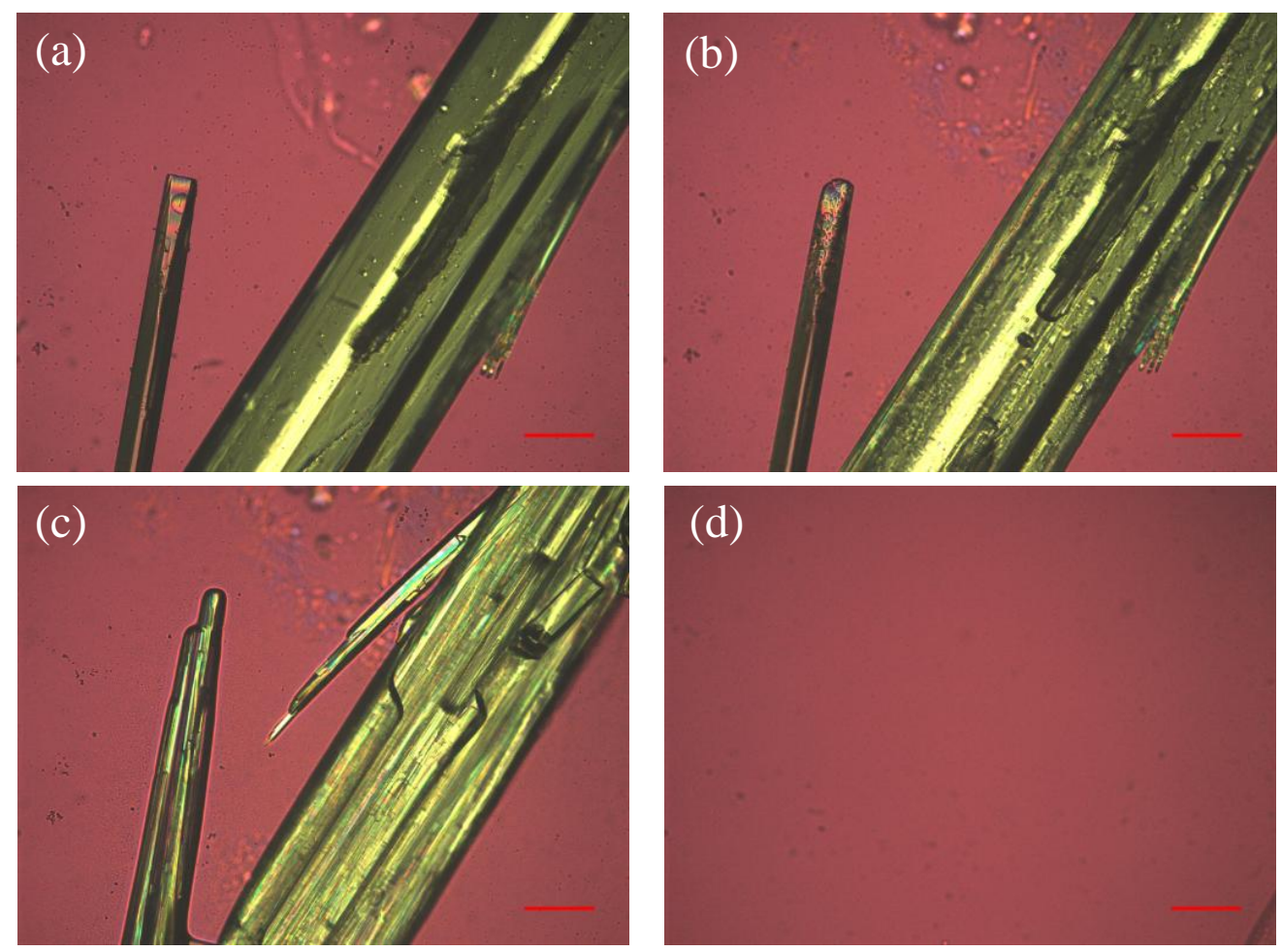

Figure S13. POM observation of the dissociation and recrystallization of DIF-PCLdiol530 IC crystals at a heating rate of $10{ }^{\circ} \mathrm{C} / \mathrm{min}$. (a) r.t., (b) $174{ }^{\circ} \mathrm{C}$, (c) $194{ }^{\circ} \mathrm{C}$ and (d) $214{ }^{\circ} \mathrm{C}$. The bars represent $100 \mu \mathrm{m}$. 

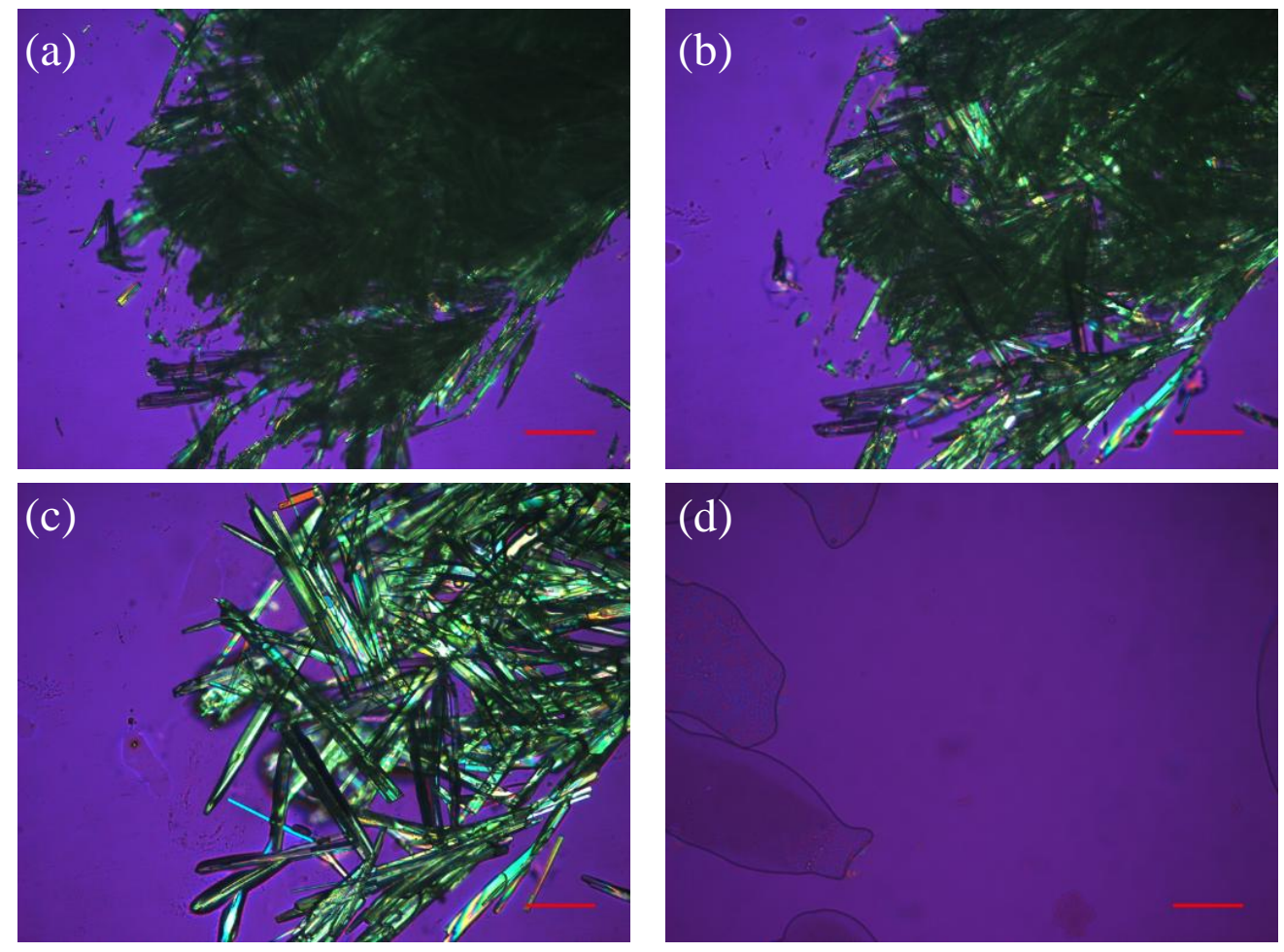

Figure S14. POM observation of the dissociation and recrystallization of DIF-PTHF2900 IC crystals at a heating rate of $10{ }^{\circ} \mathrm{C} / \mathrm{min}$. (a) r.t., (b) $170{ }^{\circ} \mathrm{C}$, (c) $180{ }^{\circ} \mathrm{C}$ and (d) $214{ }^{\circ} \mathrm{C}$. The bars represent $100 \mu \mathrm{m}$.
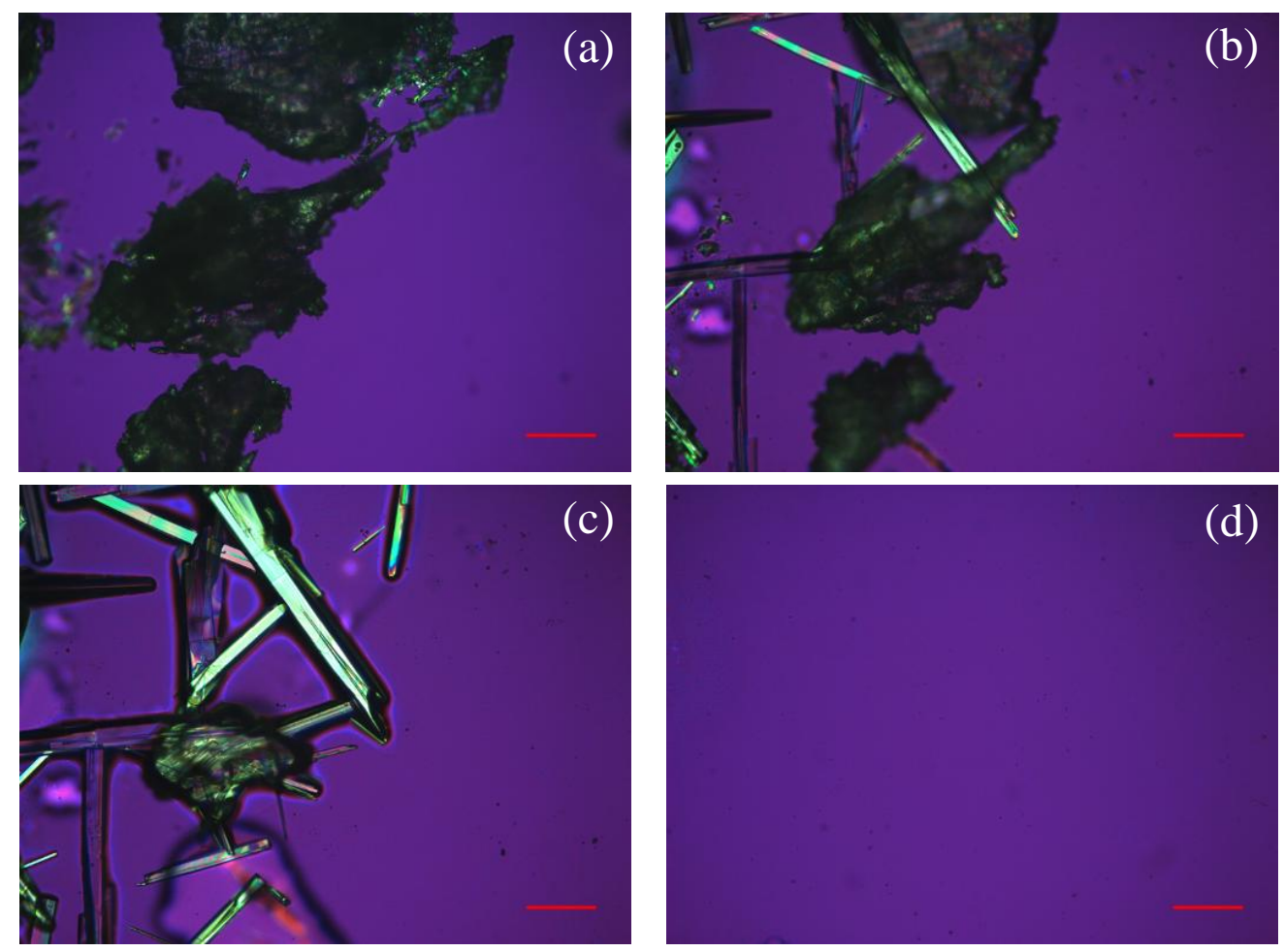

Figure S15. POM observation of the dissociation and recrystallization of DIF-PCL10000 IC crystals at a heating rate of $10{ }^{\circ} \mathrm{C} / \mathrm{min}$. (a) r.t., (b) $200{ }^{\circ} \mathrm{C}$, (c) $205^{\circ} \mathrm{C}$ and (d) $214{ }^{\circ} \mathrm{C}$. The bars represent $100 \mu \mathrm{m}$. 

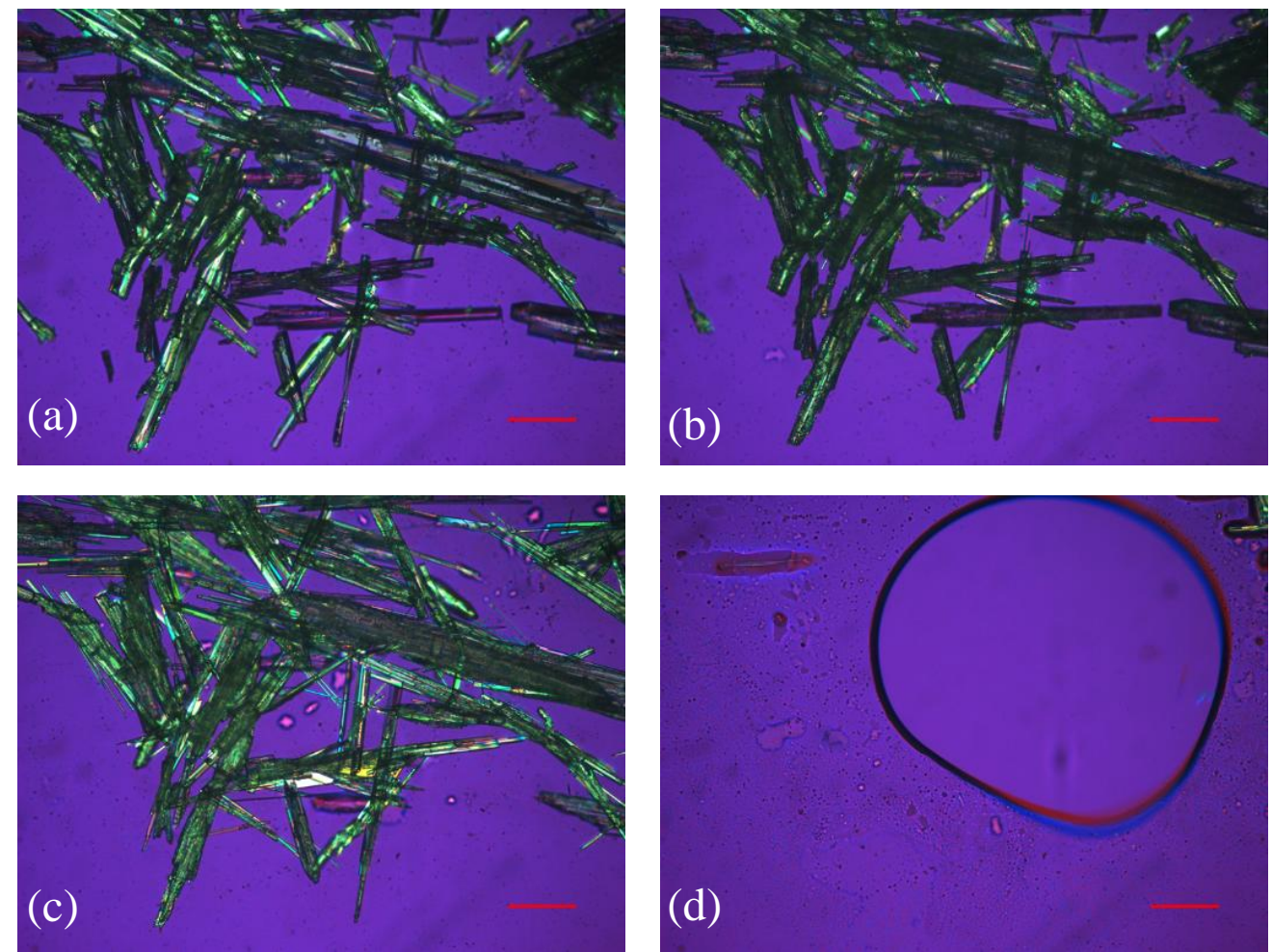

Figure S16. POM observation of the dissociation and recrystallization of DIF-P4HB4000 IC crystals at a heating rate of $10{ }^{\circ} \mathrm{C} / \mathrm{min}$. (a) r.t., (b) $172{ }^{\circ} \mathrm{C}$, (c) $188^{\circ} \mathrm{C}$ and (d) $214{ }^{\circ} \mathrm{C}$. The bars represent $100 \mu \mathrm{m}$.
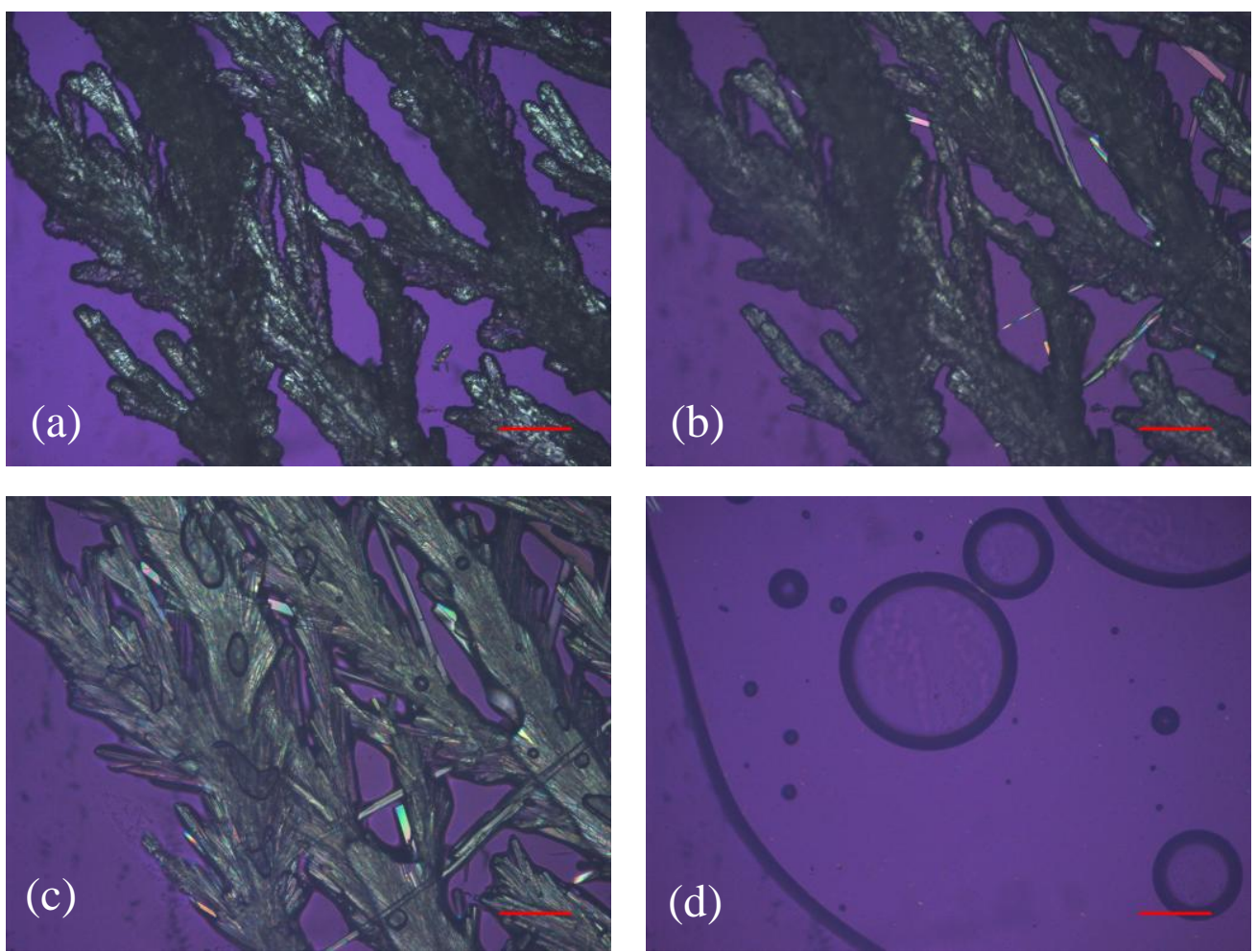

Figure S17. POM observation of dissociation and recrystallization of DIF-PBA12000 IC crystals at a heating rate of $10{ }^{\circ} \mathrm{C} / \mathrm{min}$. (a) r.t., (b) $205^{\circ} \mathrm{C}$, (c) $210{ }^{\circ} \mathrm{C}$ and (d) $214^{\circ} \mathrm{C}$. The bars represent $100 \mu \mathrm{m}$. 


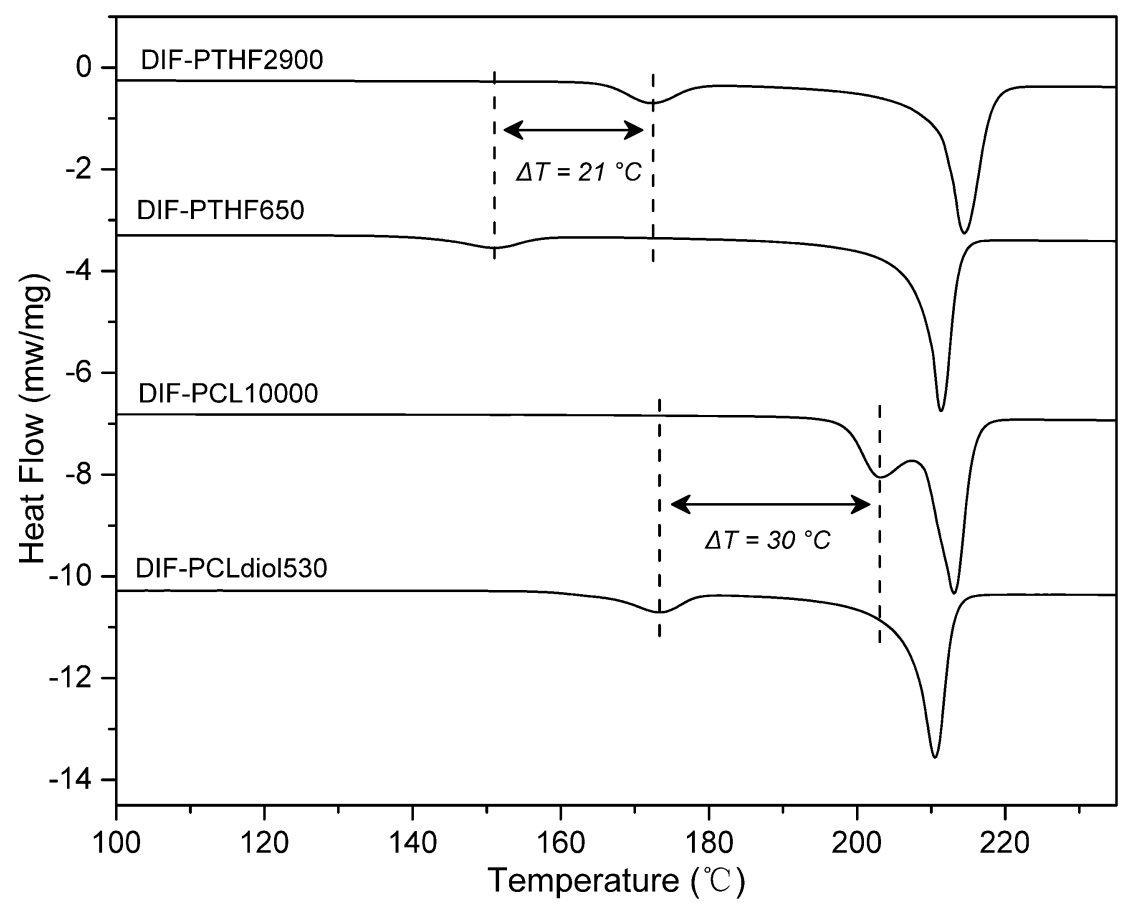

Figure S18. DSC thermograms of DIF-PTHF and DIF-PCL ICs measured at a heating rate of $10{ }^{\circ} \mathrm{C} / \mathrm{min}$. The decrease of chain length for PTHF from Mn 2900 to $M n 650$ caused a significant depression in the dissociation temperature from $172{ }^{\circ} \mathrm{C}$ to $151{ }^{\circ} \mathrm{C}$, while for DIF-PCL a larger depression from $203^{\circ} \mathrm{C}$ to $173{ }^{\circ} \mathrm{C}$ was observed.

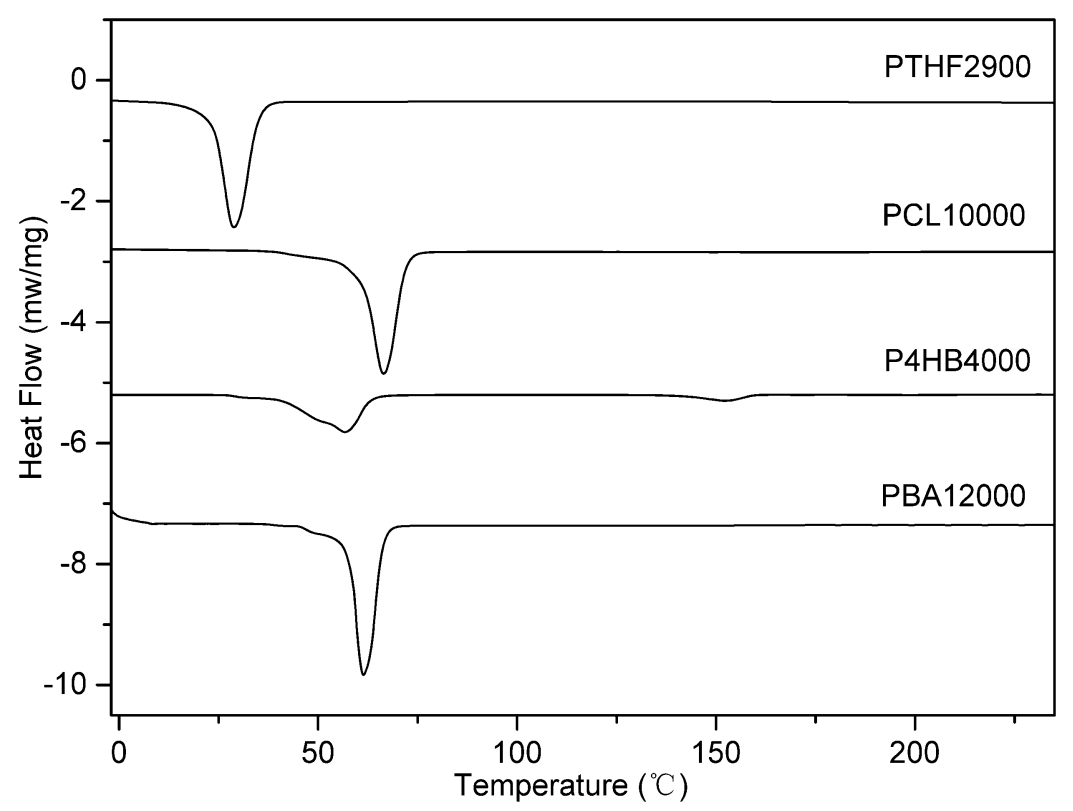

Figure S19. DSC thermograms of the polymer guest measured at a heating rate of $10{ }^{\circ} \mathrm{C} / \mathrm{min}$. The endothermal event occurred around $152{ }^{\circ} \mathrm{C}$ for P4HB sample is attributed to the melting of the $\mathrm{P} 3 \mathrm{HB}$ in the raw material. 


\section{References}

(1) (a) Martínez-Ohárriz, M. C.; Martín, C.; Goñi, M. M.; Rodríguez-Espinosa, C.; de Ilarduya-Apaolaza, M. C. Tros; Sánchez, M. J. Pharm. Sci. 1994, 83, 174. (b) Cross, W. I.; Blagden, N.; Davey, R. J.; Pritchard, R. G.;

Neumann, M. A.; Roberts, R. J.; Rowe, R. C. Cryst. Growth Des. 2003, 3, 151.

(2) Sheldrick, G. Acta Crystallogr. A 2008, 64, 112.

(3) Sheldrick, G. Acta Crystallogr. C 2015, 71, 3.

(4) Spek, A. Acta Crystallogr. D 2009, 65, 148.

(5) Hansen, L. K.; Perlovich, G. L.; Bauer-Brandl, A. Acta Crystallogr. E 2001, 57, o604.

(6) Hansen, L. K.; Perlovich, G. L.; Bauer-Brandl, A. Acta Crystallogr. E 2001, 57, o477.

(7) Macrae, C. F.; Bruno, I. J.; Chisholm, J. A.; Edgington, P. R.; McCabe, P.; Pidcock, E.; Rodriguez-Monge, L.; Taylor, R.; van de Streek, J.; Wood, P. A. J. Appl. Crystallogr. 2008, 41, 466.

(8) (a) Vogt, F. G.; Yin, H.; Forcino, R. G.; Wu, L. Mol. Pharmaceutics 2013, 10, 3433. (b) Pham, T. N.; Watson, S. A.; Edwards, A. J.; Chavda, M.; Clawson, J. S.; Strohmeier, M.; Vogt, F. G. Mol. Pharmaceutics 2010, 7, 1667.

(9) Dreyfuss, P.; Dreyfuss, M. P. Adv. Polym. Sci. 1967, 4, 528.

(10) Bittiger, H.; Marchessault, R. H.; Niegisch, W. D. Acta Crystallogr. B 1970, 26, 1923.

(11) Minke, R.; Blackwell, J. J. Macromol. Sci., Part B: Phys. 1979, 16, 407. 